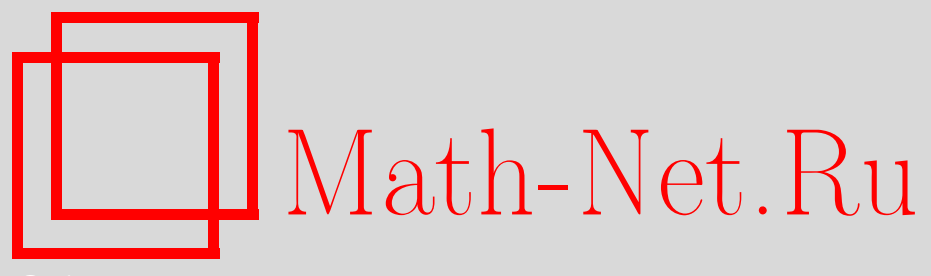

В. В. Жиков, С. Е. Пастухова, О повышенной суммируемости градиента решений эллиптических уравнений с переменным показателем нелинейности, Матем. сб., 2008, том 199, номер 12, 19-52

DOI: https://doi.org/10.4213/sm5008

Использование Общероссийского математического портала Math-Net.Ru подразумевает, что вы прочитали и согласны с пользовательским соглашением http://www.mathnet.ru/rus/agreement

Параметры загрузки:

IP: 3.81 .55 .215

26 апреля 2023 г., 14:36:05

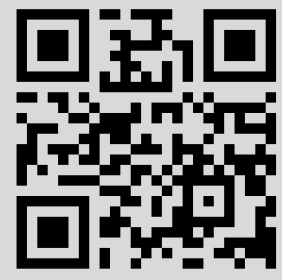




\author{
В. В. Жиков, С. Е. Пастухова
}

\title{
О повышенной суммируемости градиента решений эллиптических уравнений с переменным показателем нелинейности
}

\begin{abstract}
Изучаются эллиптические уравнения типа $p(x)$-лапласиана. Хорошо известно логарифмическое условие на модуль непрерывности показателя нелинейности $p(x)$, при котором лапласиан переменного порядка нелинейности наследует многие свойства обычного $p$-лапласиана постоянного порядка. Среди них - так называемое свойство повышенной суммируемости градиента решения. В настоящей работе установлено, что это свойство сохраняется при некотором более общем условии на показатель $p(x)$, но повышение суммируемости оказывается не степенного, а логарифмического характера. В основе предложенного метода лежит новое обобщение леммы Геринга, в котором исходным является обратное неравенство Гёльдера "с увеличенными носителем и показателем в правой части". Построен контрпример, показывающий, насколько точно найденное условие на модуль непрерывности.
\end{abstract}

Библиография: 28 названий.

\section{§ 1. Введение}

1.1. Из истории вопроса. В ограниченной области $\Omega \subset \mathbb{R}^{n}, n \geqslant 2$, рассмотрим задачу Дирихле

$$
\Delta u=\operatorname{div} h \text { в } \Omega,\left.\quad u\right|_{\partial \Omega}=0, \quad h \in L^{2}(\Omega) .
$$

Решение понимается в слабом смысле, т.е.:

$$
u \in H_{0}^{1,2}(\Omega), \quad \int_{\Omega} \nabla u \cdot \nabla \varphi d x=\int_{\Omega} h \cdot \nabla \varphi d x \quad \forall \varphi \in C_{0}^{\infty}(\Omega) .
$$

Здесь и далее через $H_{0}^{1, q}(\Omega), q>1$, обозначаем замыкание множества $C_{0}^{\infty}(\Omega)$ по норме

$$
\left(\int_{\Omega}|\nabla \varphi|^{q} d x\right)^{1 / q}
$$

По замыканию в качестве пробной функции в тождество (1.2) можно подставить само решение и получить $L^{2}$-оценку для градиента $\nabla u$ :

$$
\int_{\Omega} \nabla u \cdot \nabla u d x=\int_{\Omega} h \cdot \nabla u d x \leqslant\|h\|_{L^{2}(\Omega)}\|\nabla u\|_{L^{2}(\Omega)} \quad \Longrightarrow \quad\|\nabla u\|_{L^{2}(\Omega)} \leqslant\|h\|_{L^{2}(\Omega)} .
$$


Возникает следующий вопрос о возможной повышенной суммируемости градиента решения: если $h \in L^{q}(\Omega), q>2$, то будет ли верна аналогичная $L^{q}$-оценка для $\nabla u$ ? Положительный ответ дает теорема Кальдерона-Зигмунда [1], согласно которой

$$
\|\nabla u\|_{L^{q}(\Omega)} \leqslant c(\Omega, q)\|h\|_{L^{q}(\Omega)} \quad \forall q>2,
$$

если область $\Omega$ достаточно регулярна. Будем говорить, что область $\Omega$ из класса $D^{q}, q \in(2, \infty)$, если для заданного $q$ верна оценка (1.4).

Теперь возьмем вместо (1.1) равномерно эллиптическое уравнение

$$
\operatorname{div}(a(x) \nabla u)=\operatorname{div} h, \quad x \in \Omega,\left.\quad u\right|_{\partial \Omega}=0, \quad h \in L^{2}(\Omega),
$$

с измеримой симметричной матрицей $a(x)$, подчиненной условию

$$
\lambda|\xi|^{2} \leqslant a(x) \xi \cdot \xi \leqslant|\xi|^{2}, \quad 0<\lambda<1,
$$

для всех $\xi \in \mathbb{R}^{n}$ и п.в. $x \in \Omega$. Для задачи (1.5), как и выше, легко вывести $L^{2}$-оценку

$$
\|\nabla u\|_{L^{2}(\Omega)} \leqslant \frac{1}{\lambda}\|h\|_{L^{2}(\Omega)}
$$

с константой, не зависящей от области $\Omega$, а свойство повышенной суммируемости градиента решения также сохраняется, но в форме, ограниченной по сравнению с (1.4).

ТеОрема (Мейерс, см. [2]). Пусть область $\Omega$ класса $D^{q}$ для некоторого $q>2$ и и - решение задачи (1.5). Тогда найдется $p_{0} \in(2, q)$, зависящее лишь от константы эллиптичности $\lambda$ и области $\Omega$, такое, что

$$
\|\nabla u\|_{L^{p}(\Omega)} \leqslant c\|h\|_{L^{p}(\Omega)}, \quad c=c(\lambda, \Omega, p) \quad \forall p \in\left(2, p_{0}\right) .
$$

Мейерс доказал неравенство (1.7), представляя уравнение (1.5) как возмущение лапласиана и опираясь на оценку Кальдерона-Зигмунда, а также на интерполяционную теорему Рисса-Торина для $L^{p}$-пространств. Идея использовать метод возмущения заимствована из работы Б. Боярского [3], где подобные вопросы изучались для эллиптических систем уравнений первого порядка с измеримыми коэффициентами, но только на плоскости и ограничение размерности $n=2$ было существенно.

Чтобы объяснить, откуда в оценке (1.7) возникает ограничение на показатель $p$, напомним некоторые моменты из доказательства теоремы Мейерса. С уравнениями (1.1) и (1.5) свяжем определенные на произведении $H_{0}^{1, p}(\Omega) \times$ $H_{0}^{1, p^{\prime}}(\Omega), p^{\prime}=p /(p-1), 2 \leqslant p \leqslant q$, билинейные формы

$$
\mathscr{B}(v, w)=\int_{\Omega} \nabla v \cdot \nabla w d x, \quad \mathscr{B}_{a}(v, w)=\int_{\Omega} a \nabla v \cdot \nabla w d x .
$$

Положим

$$
\inf _{\|\nabla w\|_{L^{p^{\prime}}(\Omega)}=1} \sup _{\|\nabla v\|_{L^{p}(\Omega)}=1}|\mathscr{B}(v, w)|=\frac{1}{k_{p}} .
$$


Из энергетического неравенства (1.3) и оценки Кальдерона-Зигмунда с помощью интерполяционных соображений можно получить следующие свойства константы $k_{p}: \min _{p} k_{p}=k_{2}=1, k_{p}$ непрерывна и не убывает при $p \geqslant 2$.

Теперь обратимся к форме $\mathscr{B}_{a}$. Из условия (1.6) выводим оценку

$$
\inf _{\|\nabla w\|_{L^{p^{\prime}(\Omega)}}=1} \sup _{\|\nabla v\|_{L^{p}(\Omega)}=1}\left|\mathscr{B}_{a}(v, w)\right| \geqslant \frac{1}{k_{p}}-1+\lambda \equiv k(p),
$$

которая оказывается весьма значимой благодаря двум обстоятельствам. Вопервых, доказано, что в случае $k(p)>0$ уравнение $(1.5)$ разрешимо в $H_{0}^{1, p}(\Omega)$ для $h \in L^{p}(\Omega)$ с оценкой $(1.7)$, в которой $c=1 / k(p)$. Во-вторых, величина $k(p)$ из (1.8) в самом деле положительна при $p$, достаточно близких к значению 2 в силу перечисленных свойств константы $k_{p}$. Когда $k(p)$ становится отрицательной при $p>p_{0}$, появляется граница для показателя $p$ в $L^{p}$-оценке, и данная ситуация действительно реализуется.

ПримеР (Мейерс, см. [2]). Пусть $n=2, \Omega=\left\{x=\left(x_{1}, x_{2}\right) \in \mathbb{R}^{2}|| x \mid<1\right\}$, $a_{11}(x)=1+\left(\mu^{2}-1\right) x_{2}^{2}|x|^{-2}, a_{12}(x)=\left(1-\mu^{2}\right) x_{1} x_{2}|x|^{-2}, a_{22}(x)=1+\left(\mu^{2}-1\right) x_{1}^{2}|x|^{-2}$, $u_{0}=x_{1}|x|^{(\mu-1) / 2}$, где $\mu \in(0,1)$.

Прямыми вычислениями показывается, что $\operatorname{div}\left(a \nabla u_{0}\right)=0$, а числа 1 и $\mu$ являются собственными значениями матрицы $a(x)$ в каждой точке $x$, что обеспечивает условие (1.6) с $\lambda=\mu^{2}$. Полагая $u(x)=u_{0}(x)-x_{1}$, получаем решение задачи (1.5) с $h \in L^{\infty}(\Omega)$, при этом $\nabla u \in L^{p}(\Omega)$ для $p<2 /(1-\mu)$ и $\int_{\Omega}|\nabla u|^{2 /(1-\mu)} d x=\infty$ из-за особенности в нуле.

Геринг предложил иной подход к вопросам повышенной суммируемости. В работе [4], посвященной свойствам квазиконформных отображений, доказано следующее

УтвеРЖДЕНИЕ. Если $f \geqslant 0, f \in L_{\mathrm{loc}}^{q}(\Omega)$, где $q \in(1, \infty), \Omega$ - ограниченная область в $\mathbb{R}^{n} u$

$$
\frac{1}{|Q|} \int_{Q} f^{q} d x \leqslant c_{0}\left(\frac{1}{|Q|} \int_{Q} f d x\right)^{q}, \quad c_{0}>1,
$$

для любого куба $Q=Q_{r}\left(x_{0}\right) \subset \Omega$, где $Q_{r}\left(x_{0}\right)=x_{0}+[-r, r]^{n}$, mо

$$
f \in L_{\mathrm{loc}}^{q+\delta}(\Omega), \quad \delta=\delta\left(n, q, c_{0}\right)>0 .
$$

Таким образом, выполнение "обратного неравенства Гёльдера" (1.9) дает повышение суммируемости. Впоследствии Джаквинта и Модика [5] обобщили результат Геринга на случай, когда в левой части неравенства (1.9) среднее берется по половинному кубу $Q / 2=Q_{r / 2}\left(x_{0}\right)$. В таком виде обратное неравенство Гёльдера может быть проверено для градиента решения многих вариационных задач, что делается с помощью подходящих неравенств Каччопполи и Соболева-Пуанкаре. Разработанная в [5] общая схема приложения леммы Геринга оказалась применимой для широкого класса дифференциальных уравнений в частных производных, линейных и нелинейных [6]-[8], в том числе с переменным порядком нелинейности [9], [10]. Продолжая исследования, начатые 
в [9], [10], мы изучаем эллиптические уравнения типа $p(x)$-лапласиана при более общем условии на модуль непрерывности показателя $p(x)$, когда известные варианты леммы Геринга неприемлемы. Таким образом, потребовалось прежде всего получить подходящее обобщение этой леммы.

1.2. Обобщение леммы Геринга. Пусть $\Omega$ - область в $\mathbb{R}^{n}$ (например, $\left.\Omega=\mathbb{R}^{n}\right), f, g$ - неотрицательные функции из пространства $L^{q}(\Omega), q>1$. Считаем $f$ и $g$ продолженными нулем на все $\mathbb{R}^{n}$. Предположим, что найдутся такие $c_{0}>0, r_{0}>0$, что при $r \leqslant r_{0}$

$$
f_{Q_{r}\left(x_{0}\right)} f^{q} d x \leqslant c_{0}\left(f_{Q_{2 r}\left(x_{0}\right)} f d x\right)^{q+b(r)}+f_{Q_{2 r}\left(x_{0}\right)} g^{q} d x \quad \forall x_{0} \in \bar{\Omega},
$$

где $b(r)$ - некоторая непрерывная неубывающая функция на отрезке $\left[0, r_{0}\right]$, $b(0)=0, b(r) \leqslant 1$. Здесь и далее используем обозначение

$$
f_{E} \cdot d x=\frac{1}{|E|} \int_{E} \cdot d x-\text { среднее по множеству } E,
$$

где $|E|-n$-мерная мера Лебега измеримого множества $E$. Функцию $f$ считаем нормированной условием $\|f\|_{L^{q}} \leqslant 1$.

ТЕОРема 1.1. Если $b(r) \equiv 0$, то верна оценка

$$
\int_{\Omega} f^{q+\delta} d x \leqslant c\left(1+\int_{\Omega} g^{q+\delta} d x\right)
$$

с некоторыми с $>0, \delta>0$, зависящими только от $c_{0}, n, q, r_{0}$.

Этот результат хорошо известнен.

TEOPEMA 1.2. Пycms

$$
b(r)=\frac{k}{\ln (1 / r)}, \quad k>0 .
$$

Тогда верна оценка (1.11), в которой константы с $>0, \delta>0$ зависят, помимо прочего, еще и от множителя $k$.

TEOPEMA 1.3. Пусmь

$$
b(r)=\frac{k \ln \ln (1 / r)}{\ln (1 / r)}, \quad k>0 .
$$

Тогда при $k \leqslant 1 / n$ верна оченка

$$
\int_{\Omega} f^{q} \ln ^{\delta}(f+2) d x \leqslant c\left(1+\int_{\Omega} g^{q} \ln ^{\delta}(g+2) d x\right)
$$

с некоторыми $\delta>0, c>0$, зависящими только от $c_{0}, n, q, r_{0}$.

По поводу теорем 1.1-1.3 сделаем несколько замечаний. 
ЗАмЕчаниЕ 1.4. Обсудим имеющееся в теореме 1.3 условие $k \leqslant 1 / n$. Могло бы показаться, что при $k>1 / n$ повышенная суммируемость сохраняется, только вместо $\ln ^{\delta}(s+2)$ надо взять какую-то другую неограниченно растущую при $s \rightarrow \infty$ функцию. Однако это не так. Установлено, что при $k>3 / n$ отсутствует оценка вида (1.12) с какой-либо неограниченно растущей при $s \rightarrow \infty$ функцией вместо $\ln ^{\delta}(s+2)$ (см. $\left.\S 5\right)$.

ЗАмечАниЕ 1.5. Пусть $\Omega$ - ограниченная липшицева область. Тогда оценка (1.10) следует из неравенства

$$
f_{\Omega_{r}\left(x_{0}\right)} f^{q} d x \leqslant c_{0}\left(f_{\Omega_{2 r}\left(x_{0}\right)} f d x\right)^{q+b(r)}+f_{\Omega_{2 r}\left(x_{0}\right)} g^{q} d x, \quad x_{0} \in \bar{\Omega}
$$

в котором $\Omega_{r}\left(x_{0}\right)=\Omega \cap Q_{r}\left(x_{0}\right)$. Таким образом, для краевых задач в ограниченной липшицевой области можно проверять в качестве исходного вместо (1.10) неравенство (1.13), что иногда оказывается более естественным (см. §3).

ЗАмечАниЕ 1.6. Теоремы 1.1-1.3, применимые к произвольным областям $\Omega$, как и их аналоги для $\mathbb{R}^{n}$ - теоремы $6.1-6.3$, выводятся из одного общего варианта леммы Геринга, доказанного авторами в работе [11] - теоремы 6.4. В этом обобщении классического результата Геринга неравенство (1.10) допускает функции $b(r)$ весьма общего вида. Для удобства чтения приводим доказательство общей теоремы, а также ее следствий в $§ 6$.

ЗАмЕчАНИЕ 1.7. В исходном неравенстве (1.10) можно было бы использовать не кубы $Q_{r}, Q_{2 r}$, а шары $B_{r / 2}, B_{r}$. Формулировка в терминах шаров иногда более удобна для приложений (см. §3).

\section{§ 2. Формулировка основных результатов}

В ограниченной липшицевой области $\Omega \subset \mathbb{R}^{n}$ рассмотрим задачу Дирихле

$$
\operatorname{div}\left(|\nabla u|^{p(x)-2} \nabla u\right)=\operatorname{div} h,\left.\quad u\right|_{\partial \Omega}=0 .
$$

Предполагаем, что $h \in L^{\infty}(\Omega)$, а измеримый показатель $p(x)$ удовлетворяет условию

$$
1<\alpha \leqslant p(x) \leqslant \beta<\infty .
$$

Сначала уточним, как мы понимаем решение задачи (2.1). Если показатель $p$ постоянен, задача однозначно разрешима в соболевском пространстве $W_{0}^{1, p}(\Omega)$ (см. [12]). В случае переменного $p$ решение ищем в пространстве Соболева-Орлича

$$
W=W_{0}^{1, p(\cdot)}(\Omega)=\left\{u \in W_{0}^{1,1}(\Omega), \int_{\Omega}|\nabla u|^{p(x)} d x<\infty\right\},
$$

которое можно наделить нормой и получить сепарабельное рефлексивное банахово пространство (см. подробнее в $\S 7$ ). Будем говорить, что $u \in W$ есть 
решение задачи Дирихле (2.1), если интегральное тождество

$$
\int_{\Omega}|\nabla u|^{p-2} \nabla u \cdot \nabla \varphi d x=\int_{\Omega} h \cdot \nabla \varphi d x
$$

выполнено для любой пробной функции $\varphi \in W$. В указанном смысле задача (2.1) однозначно разрешима, что можно доказать с помощью вариационных соображений или техники монотонных операторов [9], [10], [13], действуя так, будто показатель $p$ постоянен. Однако в случае переменного показателя $p$ есть одно существенное отличие, связанное с тем, что гладкие функции, вообще говоря, не плотны в пространстве $W: H \neq W$, где $H$ - замыкание множества $C_{0}^{\infty}(\Omega)$ в $W$. Поэтому наряду с введенным выше $W$-решением можно определить $H$-решение как такую функцию $u \in H$, для которой интегральное тождество (2.3) выполнено для любой $\varphi \in H$. В обоих случаях в тождестве (2.3) в качестве пробной функции можно взять само решение и получить энергетическое равенство и оценку

$$
\int_{\Omega}|\nabla u|^{p} d x=\int_{\Omega} h \cdot \nabla u d x \quad \Longrightarrow \quad \int_{\Omega}|\nabla u|^{p} d x \leqslant 2^{\alpha^{\prime}+1} \int_{\Omega}|h|^{p^{\prime}} d x .
$$

Здесь "штрих" указывает на сопряженный по Гёльдеру показатель, например $\alpha^{\prime}=\alpha /(\alpha-1)$. В случае равенства $H=W$ показатель $p$ и саму задачу Дирихле называют регулярными. Важно знать условия регулярности. Для непрерывного показателя $p(x)$ свойство регулярности зависит от его модуля непрерывности $\omega(t)$. Напомним, что по определению модуля непрерывности

$$
|p(x)-p(y)| \leqslant \omega(|x-y|), \quad x, y \in \Omega, \quad|x-y| \leqslant \frac{1}{4} .
$$

Хорошо известно следующее логарифмическое условие: если

$$
\omega(t)=\frac{k}{\ln (1 / t)}, \quad k>0,
$$

то показатель $p$ регулярен [14]. Более того, это условие влечет множество других свойств: гёльдеровость решения, повышенную суммируемость градиента, ограниченность максимального оператора Харди-Литтлвуда в пространстве Орлича и др. (см. [15]-[19].

Сравнительно недавно [15] было установлено, что для модуля непрерывности

$$
\omega(t)=\frac{k_{0} \ln \ln (1 / t)}{\ln (1 / t)}, \quad k_{0}>0
$$

также имеет место регулярность, если только $k_{0} \leqslant \alpha / n$, а при $k_{0}>\alpha / n$ уже строятся примеры, когда $H \neq W$. Однако помимо регулярности каких-либо других свойств при $k_{0} \leqslant \alpha / n$ до сих пор доказано не было. Обобщение леммы Геринга в форме теоремы 1.3 позволяет и в этом случае получить некоторое свойство повышенной суммируемости градиента. 
ТЕОРема 2.1. Пусть показатель $p(x)$ имеет модуль непреръвности (2.6), причем $k_{0}<\alpha / n$. Тогда для решения задачи (2.1) верна ощенка

$$
\int_{\Omega}|\nabla u|^{p} \ln ^{\delta}(|\nabla u|+2) d x \leqslant c \int_{\Omega}|h|^{p^{\prime}} \ln ^{\delta}(|h|+2) d x+c,
$$

где $\delta>0, c>0$ зависят только от $\alpha, \Omega, n$.

Для сравнения заметим, что в случае логарифмического модуля непрерывности (2.5) наблюдается степенное повышение суммируемости градиента, а именно

$$
\int_{\Omega}|\nabla u|^{(1+\delta) p} d x \leqslant c \int_{\Omega}|h|^{(1+\delta) p^{\prime}} d x+c,
$$

где $\delta>0, c>0$ зависят только от $\Omega, \alpha, n, k$. Этот результат впервые доказан в [9], [10] (см. также [15]).

Теорема 2.1 теряет силу при $k_{0}>3 \alpha / n$. Более того, в этом случае, вообще говоря, отсутствует оценка вида (2.7) с какой-либо неограниченно растущей функцией вместо $\ln ^{\delta}(s+2)$. Доказательство состоит в построении гладких показателей $p_{\varepsilon}$ таких, что

(i) $1<\alpha \leqslant p_{\varepsilon} \leqslant \beta$;

(ii) $p_{\varepsilon}$ имеют общий модуль непрерывности (2.6) с некоторым $k_{0}>3 \alpha / n$;

(iii) в уравнении вида (2.1) с $p=p_{\varepsilon}$ вектор $h \in\left(L^{\infty}\right)^{n}$ не зависит от $\varepsilon$;

(iv) последовательность $\left|\nabla u_{\varepsilon}\right|^{p_{\varepsilon}}$ не является слабо компактной в $L^{1}(\Omega)$.

Построение проделано в $\S 5$ на основе модельного примера из $\S 4$. Множитель $k_{0}$ можно взять сколь угодно близким к $3 \alpha / n$. В случае $k_{0} \in[\alpha / n, 3 \alpha / n]$ положение осталось неясным.

Для $p(x)$-лапласиана мы рассматриваем также краевые задачи Неймана и задачи смешанного типа, которые ставятся соответственно в пространстве Соболева-Орлича

$$
W^{1, p(\cdot)}(\Omega)=\left\{u \in W^{1,1}(\Omega), \int_{\Omega}|\nabla u|^{p(x)} d x<\infty\right\}
$$

или его подпространстве функций с нулевым следом на выделенной части границы $\partial \Omega$. Если показатель $p(x)$ имеет модуль непрерывности вида $(2.5)$ или (2.6), решение этих задач также обладает свойством повышенной суммируемости градиента типа (2.8) или (2.7) (подробности см. в §3).

\section{§ 3. Свойство повышенной суммируемости градиента}

3.1. Рассмотрим задачу Дирихле в ограниченной липшицевой области $\Omega \subset$ $\mathbb{R}^{n}, n \geqslant 2$ :

$$
u \in W_{0}^{1, p(\cdot)}(\Omega), \quad \operatorname{div}\left(|\nabla u|^{p(x)-2} \nabla u\right)=\operatorname{div} h, \quad x \in \Omega,
$$

где $h \in L^{p^{\prime}(\cdot)}(\Omega)^{n}$. Решение этой задачи мы понимаем в смысле интегрального тождества

$$
\int_{\Omega}\left(|\nabla u|^{p(x)-2} \nabla u-h\right) \cdot \nabla \varphi d x=0 \quad \forall \varphi \in W_{0}^{1, p(\cdot)}(\Omega) .
$$


Таким образом, мы выделяем $W$-решение задачи Дирихле (3.1). Можно было бы взять и $H$-решение этой задачи (см. о краевых задачах двух типов в 2 ) дальнейшие выводы справедливы для решений обоих типов. Впрочем, когда речь пойдет непосредственно о свойстве повышенной суммируемости градиента, $W$ - и $H$-решение совпадают.

$1^{\circ}$. Неравенство Каччопполи. Пусть показатель $p(x)$ непрерывен в $\bar{\Omega}$ и выполнено условие $(2.2)$, а решение $u$ и правая часть $h$ продолжены нулем вне области $\Omega$.

ЛЕмма 3.1. Если и - решение задачи (3.1), то выполнено неравенство

$$
\int_{B_{r / 2}\left(x_{0}\right)}|\nabla u|^{p(x)} d x \leqslant c \int_{B_{r}\left(x_{0}\right)}\left(\left|\frac{u-\lambda}{r}\right|^{p(x)}+|h|^{p^{\prime}(x)}\right) d x, \quad c=c(\beta),
$$

для любого шара $B_{r}=B_{r}\left(x_{0}\right)=\left\{x|| x-x_{0} \mid<r\right\}$ с иентром в точке $x_{0} \in \bar{\Omega}$. Здесь

$$
\lambda=\left\{\begin{array}{ll}
f_{B_{r}} u d x, & \text { если } B_{3 r / 4} \subset \Omega, \\
0 & \text { в противном случае. }
\end{array} .\right.
$$

ДокАзАТЕльство. В случае непрерывного $p(x)$ верен принцип локализации (cм. §6):

$$
u \in W^{1, p(\cdot)}(\Omega), \quad \psi \in C_{0}^{\infty}(\Omega) \quad \Longrightarrow \quad \psi u \in W_{0}^{1, p}(\Omega) .
$$

Тогда легко показать, что в качестве пробной функции в тождестве (3.2) можно взять

$$
\varphi=\chi^{\beta}(u-\lambda), \quad \chi \in C_{0}^{\infty}\left(B_{3 r / 4}\right), \quad 0 \leqslant \chi \leqslant 1,\left.\quad \chi\right|_{B_{r / 2}} \equiv 1, \quad|\nabla \chi| \leqslant 5 r^{-1} .
$$

Отсюда получаем оценку

$$
\int_{B_{r}}|\nabla u|^{p(x)} \chi^{\beta} d x \leqslant 5 \beta \int_{B_{r}} \chi^{\beta-1}\left(|\nabla u|^{p(x)-1}+|h|\right)\left|\frac{u-\lambda}{r}\right| d x+\int_{B_{r}} h \chi^{\beta} \cdot \nabla u d x .
$$

По неравенству Юнга при $\varepsilon \in(0,1)$ имеем

$\chi^{\beta-1}|\nabla u|^{p-1}\left|\frac{u-\lambda}{r}\right| \leqslant \varepsilon^{p^{\prime}} \chi^{(\beta-1) p^{\prime}}|\nabla u|^{p}+\varepsilon^{-p}\left|\frac{u-\lambda}{r}\right|^{p} \leqslant \varepsilon^{\beta^{\prime}} \chi^{\beta}|\nabla u|^{p}+\varepsilon^{-\beta}\left|\frac{u-\lambda}{r}\right|^{p}$,

так как $p^{\prime}>\beta^{\prime},(\beta-1) p^{\prime} \geqslant(\beta-1) \beta^{\prime}=\beta$. Аналогично

$$
\left|h \chi^{\beta} \cdot \nabla u\right| \leqslant \varepsilon \chi^{\beta}|\nabla u|^{p}+\varepsilon^{-1} \chi^{\beta}|h|^{p^{\prime}}, \quad\left|h \frac{u-\lambda}{r}\right| \leqslant|h|^{p^{\prime}}+\left|\frac{u-\lambda}{r}\right|^{p} .
$$

Выбирая $\varepsilon$ достаточно малым, из (3.6)-(3.8) получаем неравенство (3.3).

$2^{\circ}$. Обобщенное неравенство Соболева-Пуанкаре. Напомним сначала стандартное неравенство Соболева-Пуанкаре в шаре $B_{r}$ : если

$$
u \in W^{1,1}\left(B_{r}\right), \quad f_{B_{r}} u d x=\lambda,
$$


TO

$$
\begin{gathered}
f_{B_{r}}\left|\frac{u-\lambda}{r}\right|^{s t} d x \leqslant c_{0}\left(f_{B_{r}}|\nabla u|^{s} d x\right)^{t} \quad \forall s \geqslant 1, \\
t \in\left[1, \frac{n}{n-1}\right], \quad c_{0}=\operatorname{const}(n, s) .
\end{gathered}
$$

Аналогичное неравенство верно, если $\lambda=0$ и $\left.u\right|_{K}=0, K \subset B_{r},|K|\left|B_{r}\right|^{-1} \geqslant \nu>0$. В последнем случае $c_{0}$ зависит также от величины $\nu$, но не от конфигурации множества $K$. Это следует, например, из [20; гл. II, лемма А.13].

Неравенство (3.9) для всех $s$ таких, что $1 \leqslant s \leqslant \beta<\infty$, допускает единую константу $c_{0}=\operatorname{const}(n, \beta)=c(n) \beta^{*} / l^{*}$, где $l^{*}-\operatorname{coпряженный~к~} l$ по Соболеву (показатель), а $c(n)$ - константа из неравенства (3.9) при $s=1$ (см. доказательство леммы А.10 в [20; гл. II]).

Наша цель - получить неравенство, подобное (3.9), для переменного показателя $p(x)$ с модулем непрерывности $\omega(t)$. Удобно выбрать $\omega(t)$ так, чтобы $|p(x)-p(y)| \leqslant \omega(|x-y| / 2)$.

Лемма 3.2. Для $u \in W^{1,1}\left(B_{r}\right)$ и оговоренного выше значения $\lambda$ выполнено неравенство

$$
\begin{gathered}
f_{B_{r}\left(x_{0}\right)}\left|\frac{u-\lambda}{r}\right|^{p(x)} d x \leqslant c+c\left(f_{B_{r}\left(x_{0}\right)}|\nabla u|^{p(x) / q} d x\right)^{q+q \omega(r) / \alpha} \forall q \in\left[1, \frac{n+1}{n}\right], \\
c=c(n, \beta),
\end{gathered}
$$

где $r \leqslant r_{0} u \omega\left(r_{0}\right) \leqslant\left(n^{2}-1\right)^{-1}$.

ДокАЗАТЕЛЬство. Полагая $p_{1}=\min _{B_{r}} p, p_{2}=\max _{B_{r}} p, t=q p_{2} / p_{1}$, согласно (3.9) получаем

$$
\begin{gathered}
f_{B_{r}}\left|\frac{u-\lambda}{r}\right|^{p(x)} d x \leqslant 1+f_{B_{r}}\left|\frac{u-\lambda}{r}\right|^{p_{2}} d x \leqslant 1+c_{0}\left(f_{B_{r}}|\nabla u|^{p_{2} / t} d x\right)^{t} \\
=1+c_{0}\left(f_{B_{r}}|\nabla u|^{p_{1} / q} d x\right)^{q p_{2} / p_{1}} \leqslant 1+c_{0}\left(1+f_{B_{r}}|\nabla u|^{p(\cdot) / q} d x\right)^{q+q \omega(r) / \alpha},
\end{gathered}
$$

где на последнем шаге мы воспользовались тем, что

$$
\frac{p_{2}}{p_{1}} q=\frac{p_{1}+\omega(r)}{p_{1}} q \leqslant q+\frac{\omega(r)}{\alpha} q .
$$

Для законного применения неравенства (3.9) осталось проверить, что

$$
t=\frac{p_{2}}{p_{1}} q \leqslant \frac{n}{n-1} .
$$

В самом деле,

$$
\frac{p_{2}}{p_{1}} q=\frac{p_{1}+\omega(r)}{p_{1}} q \leqslant\left(1+\omega\left(r_{0}\right)\right) q \leqslant \frac{n^{2}}{n^{2}-1} q \leqslant \frac{n}{n-1}
$$

для достаточно малого радиуса $r<r_{0}$, где $\omega\left(r_{0}\right) \leqslant 1 /\left(n^{2}-1\right), q \leqslant(n+1) / n$. Лемма доказана. 
Из предыдущих лемм вытекает

Лемма 3.3. Пусть и - решение задачи (3.1). Тогда найдутся постоянные $r_{0}=r_{0}(n, \omega), c_{0}=c_{0}(n, \Omega, \beta)>0$ maкue, чmо при $r<r_{0}, x_{0} \in \bar{\Omega}$

$$
\begin{gathered}
f_{B_{r / 2}\left(x_{0}\right)}|\nabla u|^{p(x)} d x \leqslant c_{0}\left(f_{B_{r}\left(x_{0}\right)}|\nabla u|^{p(x) / q} d x\right)^{q+q \omega(r) / \alpha}+c_{0} f_{B_{r}\left(x_{0}\right)}\left(1+|h|^{p^{\prime}}\right) d x \\
\forall q \in\left[1, \frac{n+1}{n}\right] .
\end{gathered}
$$

3.2. Для уравнения (3.1) в ограниченной липшицевой области $\Omega$ рассмотрим задачу Неймана, под решением которой понимаем функцию $u \in W^{1, p(\cdot)}(\Omega)$ такую, что

$$
\int_{\Omega}|\nabla u|^{p(x)-2} \nabla u \cdot \nabla \varphi d x=\int_{\Omega} h \cdot \nabla \varphi d x \quad \forall \varphi \in W^{1, p(\cdot)}(\Omega) .
$$

Мы взяли для определенности $W$-решение, а можно было - $H$-решение (см.§ 2). Вектор-функцию $h$ и поток $|\nabla u|^{p-2} \nabla u$ продолжим нулем вне $\Omega$, тем самым и плотность энергии $|\nabla u|^{p}$ продолжена нулем.

ЛЕмма 3.4. Если и-решение задачи (3.12), то найдутся константы $r_{0}=$ $r_{0}(n, \omega), c_{0}=c_{0}(n, \beta, \Omega)$ maкие, что при $r<r_{0}, x_{0} \in \bar{\Omega}$

$$
\begin{gathered}
f_{\Omega_{r / 2}\left(x_{0}\right)}|\nabla u|^{p(x)} d x \leqslant c_{0}\left[\left(f_{\Omega_{r}\left(x_{0}\right)}|\nabla u|^{p(x) / q} d x\right)^{q+q \omega(r) / \alpha}\right. \\
\left.+f_{\Omega_{r}\left(x_{0}\right)}\left(1+|h|^{p^{\prime}(x)}\right) d x\right] \quad \forall q \in\left[1, \frac{n+1}{n}\right],
\end{gathered}
$$

где $\Omega_{r}\left(x_{0}\right)=\Omega \cap B_{r}\left(x_{0}\right)$.

ДокАзАтЕльство. В тождестве (3.12) берем пробные функции вида (3.5), в которых $\lambda=f_{\Omega_{r}} u d x$. Тождество преобразуется к виду

$$
\int_{\Omega_{r}}|\nabla u|^{p} \chi^{\beta} d x=\beta \int_{\Omega_{r}} \chi^{\beta-1}\left(h-|\nabla u|^{p-2} \nabla u\right) \cdot(u-\lambda) \nabla \chi d x+\int_{\Omega_{r}} h \chi^{\beta} \cdot \nabla u d x .
$$

Отсюда так же, как при выводе леммы 3.1, получаем, что

$$
f_{\Omega_{r / 2}}|\nabla u|^{p(x)} d x \leqslant c(\beta)\left[f_{\Omega_{r}}|h|^{p^{\prime}(x)} d x+f_{\Omega_{r}}\left|\frac{u-\lambda}{r}\right|^{p(x)} d x\right] .
$$

Для оценки последнего интеграла используем следующее обобщение леммы 3.2 : если

$$
u \in W^{1, p(\cdot)}(\Omega), \quad x_{0} \in \bar{\Omega}, \quad r<r_{0}(n, \omega), \quad \lambda=f_{\Omega_{r}} u d x,
$$

Tо

$$
\begin{gathered}
f_{\Omega_{r}}\left|\frac{u-\lambda}{r}\right|^{p(x)} d x \leqslant c+c\left(f_{\Omega_{r}}|\nabla u|^{p(x) / q} d x\right)^{q+q \omega(r) / \alpha} \quad \forall q \in\left[1, \frac{n+1}{n}\right] \\
c=c(n, \beta, \Omega) .
\end{gathered}
$$


Вывод (3.16) основан на использовании неравенства

$$
\begin{gathered}
f_{\Omega_{r}}\left|\frac{u-\lambda}{r}\right|^{s t} d x \leqslant c_{0}\left(f_{\Omega_{r}}|\nabla u|^{s} d x\right)^{t} \quad \forall s \geqslant 1, \\
t \in\left[1, \frac{n}{n-1}\right], \quad c_{0}=c_{0}(n, s, \Omega),
\end{gathered}
$$

где $x_{0} \in \bar{\Omega}, \Omega_{r}=B_{r}\left(x_{0}\right) \cap \Omega, r<r_{0}$. Важно, что здесь константу $c_{0}$ можно выбрать не зависящей от $x_{0}$ и $r \leqslant r_{0}$. В самом деле, подвергая $\Omega_{r}$ растяжению, получаем подобласти $\Omega_{1}$ единичного шара $B_{1}$, для которых условие Липшица выполнено равномерно. Неравенство вида (3.17) для областей $\Omega_{1}$ можно записать с единой константой, зависящей лишь от $n, s$ и константы Липшица [20; гл. II, приложение А]. Отсюда обратной гомотетией возвращаемся к областям $\Omega_{r}$ и доказываем неравенство (3.17) для произвольного $r \leqslant r_{0}$. Далее, повторяя рассуждения из доказательства леммы 3.2 , выводим, опираясь на (3.17), неравенство (3.16).

Наконец, из (3.15), (3.16) вытекает искомая оценка (3.13). Лемма доказана.

3.3. Перейдем к непосредственному выводу свойства повышенной суммируемости градиента решения краевой задачи Дирихле или Неймана. Изложенного в предыдущих пунктах материала достаточно, чтобы применить для этой цели теоремы 1.2 и 1.3 .

Рассмотрим сначала задачу Дирихле (2.1), в которой показатель $p(x)$ имеет модуль непрерывности вида (2.6), и воспользуемся оценкой (3.11) при подходящем $q$, определенном ниже более точно. Удобно считать, что правая часть в уравнении нормирована условием (см. оценку в (2.4))

$$
2^{\alpha^{\prime}+1} \int_{\Omega}|h|^{p^{\prime}} d x=1 .
$$

Тогда исходное неравенство (1.10) для теоремы 1.3 выполнено, если положить

$$
f^{q}=|\nabla u|^{p}, \quad g^{q}=c_{0}\left(1+|h|^{p^{\prime}}\right), \quad b(r)=\frac{q k_{0}}{\alpha} \frac{\ln \ln (1 / r)}{\ln (1 / r)},
$$

а значение $q>1$ подчинить условию $q k_{0} / \alpha \leqslant 1 / n$, что возможно в силу предположения $k_{0}<\alpha / n$. Неравенство (1.12) в введенных выше обозначениях даст оценку (2.7). Теорема 2.1 доказана.

Теперь разберем случай, когда в задаче $(2.1)$ показатель $p(x)$ имеет модуль непрерывности (2.5). Полагаем $b(r)=q k /(\alpha \ln (1 / r))$, тогда в силу оценки $(3.11)$ те же, что ранее, функции $f$ и $g$ удовлетворяют исходному неравенству $(1.10)$ в условиях теоремы 1.2. В итоге получаем степенное повышение суммируемости градиента. В работах [9], [10] подобные результаты доказаны с помощью более простого варианта леммы Геринга, который приведен здесь как теоремa 1.1.

Аналогично на основе оценки (3.13) и замечания 1.5 доказывается формулируемый ниже результат о повышенной суммируемости градиента решения задачи Неймана. 
ТЕОРема 3.5. Пусть показатель $p(x)$ имеет модуль непрерывности (2.5) или (2.6) с $k_{0}<\alpha / n$. Тогда для решения задачи Неймана (3.12) с $h \in L^{\infty}(\Omega)$ верна оценка (2.8) или (2.7) соответственно.

3.4. Рассмотрим уравнение (3.1) в ограниченной липшицевой области $\Omega$ со смешанным краевым условием Дирихле-Неймана (в обобщенной постановке). После того, как в предыдущих разделах разобраны задачи Дирихле и Неймана, остановимся подробно только на новых моментах, специфичных для смешанной задачи. В остальном ход доказательства лишь намечается, но даются ссылки на уже сделанные ранее выводы.

Пусть $\Gamma$ - замкнутое подмножество границы $\partial \Omega$ ненулевой поверхностной меры,

$$
W_{\Gamma}^{1, p(\cdot)}(\Omega)=\left\{u \in W^{1, p(\cdot)}(\Omega)|u|_{\Gamma}=0\right\}
$$

Решением смешанной краевой задачи называем функцию $u \in W_{\Gamma}^{1, p(\cdot)}(\Omega)$, для которой выполнено интегральное тождество

$$
\int_{\Omega}|\nabla u|^{p(x)-2} \nabla u \cdot \nabla \varphi d x=\int_{\Omega} h \cdot \nabla \varphi d x \quad \forall \varphi \in W_{\Gamma}^{1, p(\cdot)} .
$$

При изучении смешанной задачи в общем случае неизбежны громоздкие построения и обозначения. Кроме того, не всякое взаимное расположение компонент $\Gamma$ и $\partial \Omega \backslash \Gamma$ допустимо. Для простоты и наглядности продемонстрируем наш метод в элементарном случае: $n=2, \partial \Omega$ - кривая на плоскости с прямолинейным куском $\gamma$, принадлежащим координатной оси, и тип граничного условия меняется в нескольких (двух или более) точках $A_{i}$, расположенных внутри отрезка $\gamma$. В этой ситуации удобней оперировать не шарами $B_{r}=B_{r}\left(x_{0}\right)$, а кубами (или квадратами) $Q_{r}=Q_{r}\left(x_{0}\right)=x_{0}+(-r, r)^{n}$. При замене шаров на кубы основные утверждения пп. 3.1-3.3 остаются в силе - именно в такой формулировке они далее предполагаются.

Для смешанной задачи (3.18) справедлив аналог теорем 2.1 и 3.5 и при его доказательстве ключевой является

Лемма 3.6. Оченка (3.13) остается в силе, если и-решение задачи (3.18), а вместо шаров $B_{r}\left(x_{0}\right)$ взяты кубъ $Q_{r}\left(x_{0}\right)$.

ДокАЗАТЕЛЬСтво. Каждый из допустимых кубов $Q_{r}\left(x_{0}\right)\left(x_{0} \in \bar{\Omega}, r \leqslant r_{0}\right)$ отнесем к одному из двух типов: к первому, если

$$
Q_{3 r / 4} \cap \partial \Omega \neq \varnothing, \quad\left|Q_{r} \cap \Gamma\right| \cdot\left|Q_{r} \cap \partial \Omega\right|^{-1} \geqslant \frac{1}{8},
$$

и ко второму в противном случае. Считаем $r_{0}$ столь малым, что кубы $Q_{r}$ могут содержать лишь одну “пограничную точку" $A_{i}$.

В тождестве (3.18) возьмем пробную функцию $\varphi$ из (3.5), полагая $\lambda=0$, если $Q_{r}$ первого типа, и $\lambda=f_{\Omega_{r}} f d x$, где $\Omega_{r}=Q_{r} \cap \Omega$, если $Q_{r}$ второго типа. Необходимое для пробной функции равенство $\left.\varphi\right|_{\Gamma}=0$ достигнуто за счет того, 
что либо $\lambda=0$ и $\left.u\right|_{\Gamma}=0$ для кубов первого типа, либо $\left.\chi\right|_{\Gamma}=0$ для кубов второго типа. Обрабатываем тождество (3.18) с выбранной пробной функцией $\varphi$ отдельно в каждом из возможных двух случаев.

Для кубов $Q_{r}$ второго типа, так же, как при выводе лемм 3.1 и 3.4, приходим сначала к оценке (3.15) и далее с помощью неравенства (3.16) к искомому соотношению (3.13).

Теперь рассмотрим кубы $Q_{r}$ первого типа. Из равенства (3.14) описанным выше способом выводим (аналог неравенства (3.15))

$$
f_{\Omega_{r / 2}}|\nabla u|^{p} d x \leqslant c(\beta, n)\left(f_{\Omega_{r}}|h|^{p^{\prime}} d x+f_{\Omega_{r}}\left|\frac{u}{r}\right|^{p} d x\right) .
$$

Для оценки последнего интеграла применим неравенство Соболева-Пуанкаре в следующем варианте:

$$
\begin{gathered}
f_{\Omega_{r}}\left|\frac{u}{r}\right|^{p(x)} d x \leqslant c+c\left(f_{\Omega_{r}}|\nabla u|^{p(x) / q} d x\right)^{q+\omega(r) q / \alpha} \quad \forall q \in\left[1, \frac{n+1}{n}\right], \\
c=c(n, \beta, \Omega, \Gamma),
\end{gathered}
$$

где $u \in W_{\Gamma}^{1, p(\cdot)}, x_{0} \in \bar{\Omega}, r \leqslant r_{0}, \Omega_{r}=\Omega \cap Q_{r}, Q_{r}-$ куб первого типа.

Соотношение (3.21) выводится стандартными рассуждениями (см. вывод (3.10) и (3.16)) из подходящего неравенства Соболева-Пуанкаре с постоянными показателями в области $\Omega_{r}$ (им является неравенство (3.17) с $\lambda=0$ и такими же, как в (3.21), условиями на $u$ и $\left.Q_{r}\right)$, справедливость которого, в свою очередь, устанавливаем с помощью растяжения $\Omega_{r}$ в подобласти $\Omega_{1}$ единичного куба. Условие $u \in W_{\Gamma}^{1, p(\cdot)}(\Omega)$ в случае, когда $Q_{r}(x)$ - куб первого типа, влечет за собой обращение в нуль на части границы $\Omega_{1}$, относительная доля которой во всей границе $\partial \Omega_{1}$ строго отделена от нуля. Здесь важно, что для кубов первого типа $\left|Q_{r} \cap \Gamma\right|\left|\partial \Omega_{r}\right|^{-1} \geqslant \nu>0$ в силу второго условия в (3.19). Дальнейшие подробности вывода (3.21) опускаем.

Из (3.20) и (3.21) следует искомая оценка (3.13), в которой вместо шаров взяты кубы.

\section{§ 4. Модельный пример}

4.1. Пусть $\theta, r$ - полярные координаты на плоскости и $B=B_{r_{0}}=\{x \mid$ $\left.|x|<r_{0}\right\}$ - круг достаточно малого радиуса $r_{0} \in(0,1 / 30)$. Изучим пространство Соболева-Орлича $W=W_{0}^{1, p(\cdot)}(B)$ для показателя

$$
p(x)=2+k \chi(r) \Phi(\theta), \quad k=\text { const }>1, \quad \chi(r)=\frac{\ln \ln (1 / r)}{\ln (1 / r)} .
$$

Здесь $\Phi(\theta)$ - непрерывная кусочно линейная функция со следующими свойствами:

1) $\Phi(\theta)=\Phi(\theta+\pi)$ ( $\pi$-периодичность);

2) $\Phi(\theta)=\Phi(-\theta)$ (четность); 
3) $\Phi(\theta)=-\Phi(\theta+\pi / 2)(\pi / 2$-антипериодичность $)$;

4) $|\Phi(\theta)| \leqslant 1$;

5) $\Phi(\theta) \equiv 1$ при $|\theta|<\delta \leqslant \pi / 8$.

Будем считать, что $\Phi(\theta)$ - это функция, изображенная на рис. $1,($ a). Предполагаем, что параметры $k$ и $r_{0}$ подчинены условию $k \chi\left(r_{0}\right)<1$. Тогда $p(x) \geqslant$ $\alpha>1$ (см. рис. $1,(\mathrm{~b}))$.

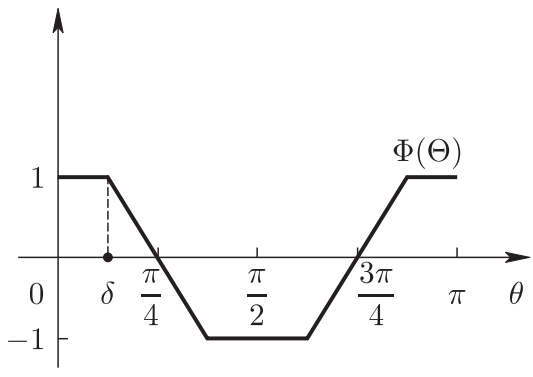

(a)

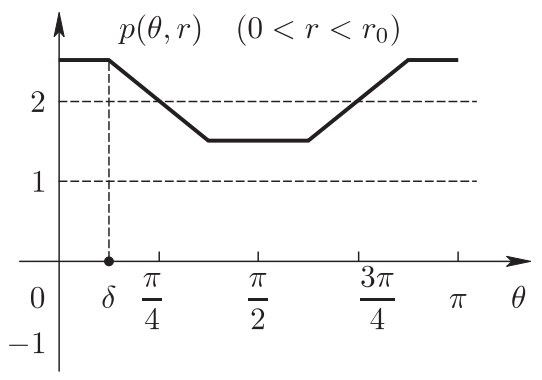

(b)

Рис. 1. Графики функций $\Phi(\cdot)$ и $p(\cdot, r)$

Показатель $p(x)$ нерегулярен, т.е. $W \neq H$, и можно утверждать большее.

Теорема 4.1. Пространство $H$ имеет коразмерность 1 в $W$.

Перед тем как приступить к доказательству этой теоремы, установим некоторые свойства функции $u \in W$. Прежде всего, в секторах $\{(\theta, r) \in B$ $|\theta+j \pi|<\delta\}, j=0,1$, где показатель $p$ принимает значение $2+k \chi(r)$ (см. рис. 2), по теореме вложения функция $u(x)$ непрерывна и определено угловое среднее

$$
\bar{u}_{j}(r)=\frac{1}{2 \delta} \int_{-\delta}^{\delta} u(\theta+j \pi, r) d \theta, \quad j=0,1
$$

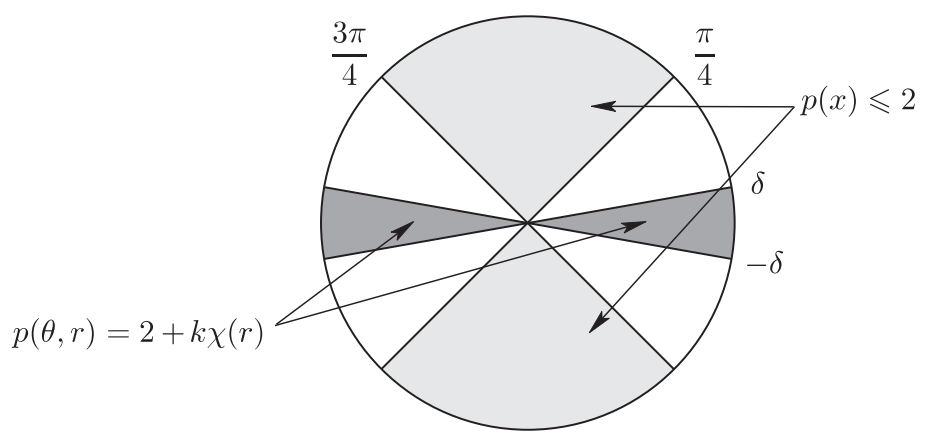

Рис. 2. Значения функции $p(x)$ в секторах круга $B$ 
ЛЕмма 4.2. Предель

$$
\lim _{r \rightarrow 0} \bar{u}_{j}(r)=c_{j}=c_{j}(u), \quad j=0,1,
$$

существуют и являются непрерывными функционалами в W. Кроме того,

$$
\bar{u}_{j}(r) \in W .
$$

ДокАЗАТЕЛЬСтво. Для определенности изучим среднее $\bar{u}(r)=\bar{u}_{0}(r)$. Вводя секторы

$$
S_{a, b}=\{(\theta, r)|| \theta \mid<\delta, a<r<b\}, \quad S_{b}=S_{0, b},
$$

можем записать

$$
\begin{gathered}
\bar{u}\left(r_{1}\right)=-\frac{1}{2 \delta} \int_{r_{1}}^{r_{0}} \int_{-\delta}^{\delta} \frac{\partial u}{\partial r} d r d \theta, \quad r_{1} \in\left(0, r_{0}\right) \\
\left|\bar{u}\left(r_{1}\right)\right| \leqslant \frac{1}{2 \delta} \int_{S_{r_{1}, r_{0}}}|\nabla u| r^{-1} d x \leqslant \frac{1}{2 \delta}\left(\int_{S_{r_{1}, r_{0}}}|\nabla u|^{p(x)} d x+\int_{S_{r_{1}, r_{0}}} r^{-p^{\prime}(x)} d x\right),
\end{gathered}
$$

где на последнем шаге использовано неравенство Юнга. Аналогично получаем

$$
\begin{gathered}
\left|\bar{u}\left(r_{1}\right)-\bar{u}\left(r_{2}\right)\right| \leqslant c\left(\int_{S_{r_{2}, r_{1}}}|\nabla u|^{p(x)} d x+\int_{S_{r_{2}, r_{1}}} r^{-p^{\prime}(x)} d x\right), \\
0<r_{2}<r_{1}<r_{0} .
\end{gathered}
$$

Из равенства

$$
p^{\prime}(x)=\frac{p(x)}{p(x)-1}=\frac{2+k \chi(r)}{1+k \chi(r)}=2-\frac{k \chi(r)}{1+k \chi(r)}
$$

следует, что $p^{\prime}(x) \leqslant 2-k_{1} \chi(r)$ в $S_{t}$ для некоторых $k_{1}>1, t \in(0,1)$. Поэтому

$$
\frac{1}{2 \delta} \int_{S_{r_{0}}} r^{-p^{\prime}(x)} d x \leqslant \int_{0}^{t} \frac{d r}{r^{1-k_{1} \chi(r)}}+\int_{S_{t, r_{0}}} r^{-p^{\prime}(x)} d x<\infty .
$$

Тогда из (4.4) заключаем, что предел $\lim _{r \rightarrow 0} \bar{u}(r)=\bar{u}(0)$ существует и является непрерывным функционалом в $W$.

Для вывода свойства (4.3) существенно постоянство функции $p(\cdot, r)$ на $(-\delta, \delta)$ (см. (4.1) и свойство 5) функции $\Phi)$. По неравенству Гёльдера

$$
\begin{gathered}
\left|\frac{\partial \bar{u}}{\partial r}\right|^{p(\theta, r)}=\left|\frac{1}{2 \delta} \int_{-\delta}^{\delta} \frac{\partial u}{\partial r}\left(\theta^{\prime}, r\right) d \theta^{\prime}\right|^{p(\theta, r)} \leqslant \frac{1}{2 \delta} \int_{-\delta}^{\delta}\left|\frac{\partial u}{\partial r}\left(\theta^{\prime}, r\right)\right|^{p(\theta, r)} d \theta^{\prime}, \\
\theta \in(-\delta, \delta),
\end{gathered}
$$

и после интегрирования по $S_{r_{0}}$ получаем

$$
\begin{aligned}
\int_{S_{r_{0}}}|\nabla \bar{u}(|x|)|^{p(x)} d x & \leqslant \frac{1}{2 \delta} \int_{-\delta}^{\delta} \int_{0}^{r_{0}} \int_{-\delta}^{\delta}\left|\frac{\partial u}{\partial r}\left(\theta^{\prime}, r\right)\right|^{p(\theta, r)} r d \theta^{\prime} d r d \theta \\
& =\int_{0}^{r_{0}} \int_{-\delta}^{\delta}\left|\frac{\partial u}{\partial r}\left(\theta^{\prime}, r\right)\right|^{p(\cdot, r)} r d \theta^{\prime} d r \leqslant \int_{S_{r_{0}}}|\nabla u|^{p(x)} d x
\end{aligned}
$$


Отсюда следует свойство $\bar{u} \in W$. В самом деле, поскольку абсолютный максимум $p(\cdot, r)$ достигается на $(-\delta, \delta)$, где эта функция постоянна, то для любой радиальной функции $f(r) \geqslant 0$ выполнено неравенство

$$
\int_{B_{r_{0}}} f(|x|)^{p(x)} d x \leqslant \frac{\pi}{\delta} \int_{S_{r_{0}}} f(|x|)^{p(x)} d x .
$$

Лемма доказана.

СлЕДСТВиЕ. Если $u \in H$, mo $_{0}(u)=c_{1}(u)$.

Действительно, для $u \in C_{0}^{\infty}(B)$ равенство $c_{0}(u)=c_{1}(u)$ выполнено, а по замыканию оно сохраняется и для $u \in H$.

Функционалы $c_{0}$ и $c_{1}$ выявляют различие между пространствами $H$ и $W$.

ЛЕмма 4.3. Для любого элемента $u \in W$ верно следующее:

$$
u \in H \quad \Longleftrightarrow \quad c_{0}(u)=c_{1}(u) \text {. }
$$

ДокАЗАТЕЛЬСтво критерия требуется провести лишь в сторону достаточности, поскольку необходимость была уже рассмотрена. Упростим ситуацию, считая $u \in L^{\infty}(B)$ (что возможно по лемме 7.5) и $c_{0}=c_{1}=0$ (что достигается вычитанием финитной гладкой функции, равной константе $c_{0}=c_{1}$ в окрестности точки $x=0)$. Разбиение единицы $\varphi_{1}(\theta)+\cdots+\varphi_{4}(\theta) \equiv 1$ на окружности позволяет ограничиться ситуацией, когда $\operatorname{supp} u$ локализован либо в одной из четвертей $\{(\theta, r) \in B|| \theta \pm \pi / 2 \mid \leqslant \pi / 4\}$, где $p \leqslant 2$ (см. (4.1) и свойства $\Phi)$, либо в одном из секторов $\{(\theta, r) \in B|| \theta-j \pi \mid<\pi / 3\}, j=0,1$, в которых определены формулой $(4.2)$ средние $\bar{u}_{j}(r)$.

Ниже мы используем следующее утверждение:

$$
\text { если } u \in W \text { и } u \equiv 0 \text { в окрестности точки } x=0 \text {, то } u \in H \text {. }
$$

Действительно, так как $u \in W_{0}^{1, p(\cdot)}(Q)$, где $Q=\{x \in B,|x|>\varepsilon\}$ - кольцо, в котором показатель $p(x)$ регулярен, то множество $C_{0}^{\infty}(Q)$ плотно в $W_{0}^{1, p(\cdot)}(Q)$, откуда следует (4.5).

Случай 1. Пусть $\operatorname{supp} u \subset\{x \mid p \leqslant 2\}$. Возьмем срезающую функцию $\eta_{\varepsilon}(x)=\eta\left(\varepsilon^{-1} x\right)$, где $\eta \in C^{\infty}\left(\mathbb{R}^{2}\right), \eta \equiv 0$ при $|x|<1 / 2, \eta \equiv 1$ при $|x|>1$, $|\nabla \eta| \leqslant 3$, и положим $u_{\varepsilon}(x)=\eta_{\varepsilon}(x) u(x)$. В силу (4.5) $u_{\varepsilon} \in H$ и достаточно доказать слабую сходимость

$$
u_{\varepsilon} \rightarrow u \text { в } W, \quad \varepsilon \rightarrow 0 .
$$

Поскольку $u_{\varepsilon} \rightarrow u$ в $L^{p(\cdot)}(B)$, то требует проверки лишь сходимость

$$
\nabla u_{\varepsilon}=\eta_{\varepsilon} \nabla u+u \nabla \eta_{\varepsilon} \rightarrow \nabla u \text { в } L^{p(\cdot)}(B), \quad \varepsilon \rightarrow 0 .
$$

Очевидно, что $\nabla u_{\varepsilon} \rightarrow \nabla u$ п.в. и по лемме 7.1 достаточно убедиться в ограниченности семейства $\nabla u_{\varepsilon}$ в $L^{p(\cdot)}$. В самом деле, $\eta_{\varepsilon} \nabla u$ ограничена в $L^{p(\cdot)}$ и по свойствам срезающей функции $\eta_{\varepsilon}$

$$
\int_{B}\left|u \nabla \eta_{\varepsilon}\right|^{p(x)} d x \leqslant C \int_{B_{\varepsilon}} \varepsilon^{-p(x)} d x \leqslant C \varepsilon^{-2}\left|B_{\varepsilon}\right|=C \pi,
$$


так как $p \leqslant 2$ на $\operatorname{supp} u$ и $u \in L^{\infty}(B)$. Таким образом, сходимости (4.7) и (4.6) получены.

Случай 2. Пусть для определенности $\operatorname{supp} u \subset\{|\theta|<\pi / 3\}$. Рассмотрим разложение

$$
u(x)=\bar{u}(r)+v(x),
$$

где $\bar{u}(r)=\bar{u}_{0}(r)$ - угловое среднее из (4.2). Считая $\bar{u}(r)$ продолженной нулем при $r<0$, введем гладкую монотонную функцию $h_{\varepsilon}(r)$ такую, что $h_{\varepsilon}(r)=\varepsilon$ при $r \leqslant \varepsilon, h_{\varepsilon}(r)=0$ при $r \geqslant 3 \varepsilon,\left|h_{\varepsilon}^{\prime}(r)\right|<1$, и положим

$$
u_{\varepsilon}(x)=\bar{u}\left(r-h_{\varepsilon}(r)\right)+\eta_{\varepsilon}(x) v(x) \equiv u_{\varepsilon}^{(1)}(x)+u_{\varepsilon}^{(2)}(x),
$$

где срезающая функция $\eta_{\varepsilon}(x)$ та же, что выше. Аппроксимация функции $u(x)$ устроена различным образом для составляющих $\bar{u}(r)$ и $v(x)$ : сдвигом в аргументе для $\bar{u}(r)$ и срезкой в окрестности точки $x=0$ для $v(x)$. В обоих случаях достигаем равенства $u_{\varepsilon}^{(i)} \equiv 0$ в круге $B_{\varepsilon}$.

Из условия $\bar{u}(r) \in W$ (см. лемму 4.2) выводим $u_{\varepsilon}^{(1)} \in W$ и далее $u_{\varepsilon}^{(1)} \in H$ (см. (4.5)). Остается доказать, что в $W$

$$
u_{\varepsilon}^{(1)}(x) \rightarrow \bar{u}(r), \quad u_{\varepsilon}^{(2)}(x) \rightarrow v(x),
$$

что дает искомое соотношение $u \in H$. Остановимся подробно лишь на ограниченности $\nabla u_{\varepsilon}^{(2)}$ в $L^{p(\cdot)}(B)$, вытекающей, как и ранее, из ограниченности $v \nabla \eta_{\varepsilon}$ в $L^{p(\cdot)}(B)($ см. $(4.8))$.

При фиксированном $r$ к функции $v(\cdot, r) / r$ на промежутке $(-\pi / 3, \pi / 3)$ применим неравенство Пуанкаре с переменным показателем $p(\cdot, r)$ (см. (7.12)), в результате чего получим, что

$$
\int_{-\pi / 3}^{\pi / 3}\left|\frac{v(\theta, r)}{r}\right|^{p(\theta, r)} d \theta \leqslant c \int_{-\pi / 3}^{\pi / 3}\left|\frac{1}{r} \frac{\partial v}{\partial \theta}\right|^{p(\theta, r)} d \theta+c .
$$

Отсюда, поскольку $v=u-\bar{u}(r),\left|\frac{1}{r} \frac{\partial v}{\partial \theta}\right| \leqslant|\nabla u|$ и $\varepsilon^{-1} \leqslant r^{-1}$ в $B_{\varepsilon}$, то

$$
\begin{aligned}
\int_{B} \mid v & \left.\nabla \eta_{\varepsilon}\right|^{p(x)} d x \leqslant c \int_{B_{\varepsilon}}\left|\frac{v}{\varepsilon}\right|^{p(x)} d x \leqslant c \int_{B_{\varepsilon}}\left|\frac{v}{r}\right|^{p(x)} d x \\
& \leqslant C \int_{0}^{\varepsilon} r\left(\int_{-\pi / 3}^{\pi / 3}\left|\frac{1}{r} \frac{\partial v}{\partial \theta}\right|^{p(\theta, r)} d \theta+1\right) d r \leqslant C\left(\int_{B_{\varepsilon}}|\nabla u|^{p(x)} d x+1\right) \\
& \leqslant C\left(\int_{B}|\nabla u|^{p(x)} d x+1\right) .
\end{aligned}
$$

Получено искомое неравенство и вместе с тем соотношение $u \in H$. Лемма доказана.

\section{2. После подготовительных лемм мы приведем}

ДокаЗАТЕЛЬСТво ТЕОРЕмы 4.1. Сначала предъявим функцию $u_{0} \in W \backslash H$. Пусть $\psi(\theta)$ - гладкая $2 \pi$-периодическая функция, $\psi(0)=0, \psi(\pi)=1,0 \leqslant \psi \leqslant 1$, $\psi^{\prime}(\theta) \equiv 0$ вне $I_{\delta}=\{\theta|| \theta \pm \pi / 2 \mid<\delta\}, \delta$ - из определения функции $\Phi(\theta)$ (см. (4.1)). 
Тогда $u_{0}(x)=\psi(\theta)\left(1-\left(r / r_{0}\right)^{2}\right) \in W$. В самом деле, ввиду ограниченности $u_{0}(x)$ требует изучения лишь градиент $\nabla u_{0}$, а точнее, его составляющая $\nabla \psi$. Поскольку $p(\cdot, r)=2-k \chi(r)$ на $I_{\delta}$ по свойству функции $\Phi(\theta)$ и $|\nabla \psi|<c r^{-1}$, то

$$
\int_{B}|\nabla \psi|^{p(x)} d x \leqslant c \int_{0}^{r_{0}} \int_{I_{\delta}} \frac{r d r d \theta}{r^{p(\theta, r)}} \leqslant 2 \delta c \int_{0}^{r_{0}} \frac{d r}{r^{1-k \chi(r)}}<\infty,
$$

что обеспечивает принадлежность $u_{0}$ пространству $W$. С другой стороны, по лемме $4.3 u_{0} \notin H$, так как $c_{0}\left(u_{0}\right)=0$ и $c_{1}\left(u_{0}\right)=1$.

Теперь возьмем произвольную функцию из $u \in W$ с предельными значениями в нуле $c_{0} \neq c_{1}$ и $\lambda=c_{1}-c_{0}$. Если $u_{0}$ - построенная выше функция, то $u-\lambda u_{0} \in H$ по лемме 4.3. Таким образом, получено представление $W=\left\{\lambda u_{0}\right\}+H$, что и требовалось.

4.3. Укажем нетривиальный функционал на $W$, равный нулю на $H$. Возьмем функции $\psi(x)$ и $u_{0}(x)=\psi(\theta)\left(1-\left(r / r_{0}\right)^{2}\right)$ из доказательства теоремы 4.1 и положим

$$
w(x)=w\left(x_{1}, x_{2}\right)=\psi\left(-x_{2}, x_{1}\right), \quad b(x)=\left\{-\frac{\partial w}{\partial x_{2}}, \frac{\partial w}{\partial x_{1}}\right\} .
$$

По построению вектор $b$ соленоидален в $B$ и принадлежит пространству Орлича $L^{p^{\prime}(\cdot)}(B)$. Отсюда следует, что функционал

$$
l(\varphi)=\int_{B} b \cdot \nabla \varphi d x
$$

непрерывен на $W$ и аннулируется на $H$. Кроме того, по построению

$$
l\left(u_{0}\right)=1
$$

В самом деле, $b(x) \cdot \nabla \psi(x) \equiv 0$ п.в. на $B$ и поэтому $b \cdot \nabla u_{0}=-b \cdot \nabla \varphi$, где $\varphi=$ $\left(r / r_{0}\right)^{2} \psi \in W^{1, \infty}(B)$. Таким образом, достаточно воспользоваться следующим предложением.

ПреДЛОЖЕНИЕ 4.4. Если $\varphi \in W^{1, \infty}(B),\left.\varphi\right|_{\partial B}=\psi, m o \int_{B} b \cdot \nabla \varphi d x=-1$.

ДоказАТЕЛЬство. Заметим, что $\nu=(\cos \theta, \sin \theta)$ - единичная внешняя нормаль к $\partial B=\left\{|x|=r_{0}\right\}$. Отсюда, учитывая свойства функций $b, \psi$ и $w$, имеем $\left.b \cdot \nu\right|_{\partial B}=-\frac{1}{r_{0}} \frac{\partial w}{\partial \theta}$ и

$$
\int_{B} \nabla \varphi \cdot b d x=\int_{\partial B} \psi b \cdot \nu d s=-\int_{|\theta-\pi|<\delta} \frac{\partial w}{\partial \theta} d \theta=-1,
$$

что и требуется.

Располагая вектором $b(x)$, построим пример задачи Дирихле, для которой $W$ - и $H$-решения не совпадают. Взяв $h=b$ в (3.1), запишем следующие тождества для $W$ - и $H$-решений:

$$
u_{1} \in W, \quad\left[u_{1}, \varphi\right]=l(\varphi) \quad \forall \varphi \in W ; \quad u_{2} \in H, \quad\left[u_{2}, \varphi\right]=l(\varphi) \quad \forall \varphi \in H,
$$


где

$$
[u, \varphi]=\int_{B}|\nabla u|^{p(x)-2} \nabla u \cdot \nabla \varphi d x .
$$

Полагая в тождествах $\varphi=u_{0}$ или $\varphi=u_{2}$, выводим $u_{1} \not \equiv 0 \equiv u_{2}$ в силу отмеченных выше свойств функционала $l:\left.l\right|_{H}=0, l\left(u_{0}\right)=1$.

По построению вектор $h=b$ принадлежит $L^{p^{\prime}(\cdot)}(B)$ и неограничен в окрестности $x=0$. Теперь при малых $\varepsilon$ возьмем срезку $h_{\varepsilon}(x)=\eta_{\varepsilon}(x) b(x) \in L^{\infty}(B)$, где $\eta_{\varepsilon}(x)=\eta(x / \varepsilon), \eta(x)=0$ в $B_{1 / 2}(0), 1-\eta(x) \in C_{0}^{\infty}\left(B_{1}(0)\right)$. Можно показать, что $\left\|h-h_{\varepsilon}\right\|_{L^{p^{\prime}(\cdot)(B)}} \rightarrow 0$ (о норме и сходимости в $L^{p^{\prime}(\cdot)}$ см. $\S 7$ ). Тогда $l_{\varepsilon}(\varphi)=\int_{B} h_{\varepsilon} \cdot \nabla \varphi d x \rightarrow l(\varphi) \forall \varphi \in W$ и, более того, $\left\|l_{\varepsilon}-l\right\|_{W^{*}} \rightarrow 0$.

Покажем, что для задачи (3.1) с правой частью $h_{\varepsilon} W$ - и $H$-решения по-прежнему не совпадают. Запишем соответствующие тождества:

$$
u_{1}^{\varepsilon} \in W, \quad\left[u_{1}^{\varepsilon}, \varphi\right]=l_{\varepsilon}(\varphi) \quad \forall \varphi \in W ; \quad u_{2}^{\varepsilon} \in H, \quad\left[u_{2}^{\varepsilon}, \varphi\right]=l_{\varepsilon}(\varphi) \quad \forall \varphi \in H .
$$

В силу первого из них имеем $\left[u_{1}^{\varepsilon}, u_{0}\right]=l_{\varepsilon}\left(u_{0}\right) \rightarrow l\left(u_{0}\right)=1$. С другой стороны, по неравенству Гёльдера (7.9)

$$
\left|\left[u_{1}^{\varepsilon}, u_{0}\right]\right| \leqslant 2\left\|\left|\nabla u_{1}^{\varepsilon}\right|^{p(x)-1}\right\|_{L^{p^{\prime}(\cdot)(B)}}\left\|\nabla u_{0}\right\|_{L^{p(\cdot)}(B)},
$$

поэтому (см. также (7.4)) $\left[u_{1}^{\varepsilon}, u_{1}^{\varepsilon}\right]>d_{0}$ для некоторого $d_{0}>0$.

Переходя к пределу в тождестве для $u_{2}^{\varepsilon}$, легко показать, что $u_{2}^{\varepsilon} \rightarrow 0$ слабо в $W$, при этом $\left[u_{2}^{\varepsilon}, u_{2}^{\varepsilon}\right]=l_{\varepsilon}\left(u_{2}^{\varepsilon}\right) \rightarrow 0$, так как $\left\|l_{\varepsilon}-l\right\|_{W^{*}} \rightarrow 0$. Отсюда $\left[u_{2}^{\varepsilon}, u_{2}^{\varepsilon}\right]<d_{0}$ при малых $\varepsilon$ и поэтому $u_{1}^{\varepsilon} \neq u_{2}^{\varepsilon}$, что и требовалось.

В частности, доказано следующее

ПреДЛОжЕНИЕ 4.5. Найдется функиия $h \in L^{\infty}(B)$, для которой $H-u W$ решения задачи (3.1) не совпадают.

4.4. Изучим свойство непрерывности самой функции $p(x)$ из (4.1). Для вычисления ее модуля непрерывности полезна следующая

Лемма 4.6. Пусть $p(x)=p(\theta, r)=\varphi(\theta) f(r)$, где $\varphi(\theta)-$ липшицева функция на единичной окружности с константой Липшица $L,|\varphi(\theta)| \leqslant 1$, a $f(r)$ непрерывная возрастающая вогнутая на $\left[0, r_{0}\right]$ функиия, $f(0)=0$. Тогда

$$
\left|p(x)-p\left(x^{\prime}\right)\right| \leqslant\left(1+\frac{L \pi}{2}\right) f\left(\left|x-x^{\prime}\right|\right) \quad \forall x, x^{\prime} \in B_{r_{0}} .
$$

ДокАЗАТЕЛЬство. Прежде всего, отметим простые следствия вогнутости:

$\left(\mathrm{C}_{1}\right) f(\lambda t) \geqslant \lambda f(t), \quad \lambda \in[0,1]$,

$\left(\mathrm{C}_{2}\right) f(t / \lambda) \leqslant(1 / \lambda) f(t), \quad \lambda \in(0,1]$,

$\left(\mathrm{C}_{3}\right) f\left(t+t^{\prime}\right) \leqslant f(t)+f\left(t^{\prime}\right)$ (субаддитивность),

$\left(\mathrm{C}_{4}\right) f(t)-f\left(t^{\prime}\right) \leqslant f\left(t-t^{\prime}\right), 0 \leqslant t^{\prime} \leqslant t$. 
Свойство $\left(\mathrm{C}_{1}\right)$ следует из определения вогнутости, так как $f(0)=0$. Свойство $\left(\mathrm{C}_{2}\right)$ получается из $\left(\mathrm{C}_{1}\right)$ заменой переменной. Для доказательства $\left(\mathrm{C}_{3}\right)$ положим $a=t+t^{\prime}, \lambda=t / a, \lambda^{\prime}=t^{\prime} / a$. Тогда по вогнутости $f(t)+f\left(t^{\prime}\right)=$ $f(\lambda a)+f\left(\lambda^{\prime} a\right) \geqslant \lambda f(a)+\lambda^{\prime} f(a)=f(a)=f\left(t+t^{\prime}\right)$, что и требуется. Свойство $\left(\mathrm{C}_{4}\right)$ есть простое следствие из $\left(\mathrm{C}_{3}\right)$.

Переходим к выводу формулы (4.9). Достаточно установить модули непрерывности $\omega_{1}(t)$ и $\omega_{2}(t)$ для функции $p(\theta, r)$ по каждой из переменных $\theta$ и $r$. Тогда общий модуль непрерывности найдем по неравенству треугольника:

$$
\begin{aligned}
\left|p(x)-p\left(x^{\prime}\right)\right| & \leqslant\left|p(x)-p\left(x^{\prime \prime}\right)\right|+\left|p\left(x^{\prime \prime}\right)+p\left(x^{\prime}\right)\right| \\
& \leqslant \omega_{1}\left(\left|x-x^{\prime \prime}\right|\right)+\omega_{2}\left(\left|x^{\prime \prime}-x^{\prime}\right|\right) \leqslant \omega_{1}\left(\left|x-x^{\prime}\right|\right)+\omega_{2}\left(\left|x^{\prime}-x\right|\right),
\end{aligned}
$$

где $\left|x^{\prime}\right|=\left|x^{\prime \prime}\right|<|x|$, а угловые координаты $x^{\prime \prime}$ и $x$ совпадают, так что $\left|x-x^{\prime \prime}\right|<$ $\left|x-x^{\prime}\right|,\left|x^{\prime \prime}-x^{\prime}\right|<\left|x-x^{\prime}\right|$.

Случай 1 . Пусть $r=|x|>\left|x^{\prime}\right|=r^{\prime}, \theta$ - общая угловая координата для $x$ и $x^{\prime}$. Тогда $\left|x-x^{\prime}\right|=r-r^{\prime}$ и поскольку $|\varphi| \leqslant 1, f(r) \geqslant f\left(r^{\prime}\right)$, то в силу свойства $\left(\mathrm{C}_{4}\right)$

$$
\left|p(x)-p\left(x^{\prime}\right)\right|=|\varphi(\theta)|\left(f(r)-f\left(r^{\prime}\right)\right) \leqslant f\left(r-r^{\prime}\right)=f\left(\left|x-x^{\prime}\right|\right) .
$$

Таким образом, радиальный модуль непрерывности равен $\omega_{1}(t)=f(t)$.

Случай 2. Пусть $|x|=\left|x^{\prime}\right|=r,\left|x-x^{\prime}\right|=\rho, \theta$ и $\theta^{\prime}$ - угловые координаты точек $x$ и $x^{\prime}$. Тогда $\rho / r=2 \sin \left(\left|\theta^{\prime}-\theta\right| / 2\right) \equiv \lambda$ и $\lambda \in[0,1]$. По липшицевости $\varphi(\theta)$ имеем

$$
\left|p(x)-p\left(x^{\prime}\right)\right| \leqslant L f(r)\left|\theta-\theta^{\prime}\right|=L \frac{\left|\theta-\theta^{\prime}\right|}{\lambda} \lambda f\left(\frac{\rho}{\lambda}\right),
$$

при этом

$$
\max _{\theta, \theta^{\prime}} \frac{\left|\theta-\theta^{\prime}\right|}{\lambda}=\max _{|\alpha| \leqslant \pi / 2} \frac{\alpha}{\sin \alpha}=\frac{\pi}{2}, \quad \lambda f\left(\frac{\rho}{\lambda}\right) \leqslant f(\rho)
$$

в силу свойства $\left(\mathrm{C}_{2}\right)$. Отсюда $\left|p(x)-p\left(x^{\prime}\right)\right| \leqslant L \frac{\pi}{2} f\left(\left|x-x^{\prime}\right|\right)$, т.е. угловой модуль непрерывности равен $\omega_{2}(t)=L \frac{\pi}{2} f(t)$. Суммируя результаты, согласно формуле (4.10) приходим к (4.9). Лемма доказана.

По лемме 4.6 модуль непрерывности показателя $p(x)$ из (4.1) равен

$$
\omega(t)=\frac{k_{0} \ln \ln (1 / t)}{\ln (1 / t)}, \quad k_{0}=k\left[1+2\left(1-\frac{4 \delta}{\pi}\right)^{-1}\right] .
$$

Поясним соотношение (4.11). Непосредственно вычислив вторую производную, убеждаемся в том, что функция $(\ln \ln (1 / t)) / \ln (1 / t)$ вогнута при $t \in(0,1 / 30)$. Легко найти константу Липшица для функции $\Phi(\theta), L=(\pi / 4-\delta)^{-1}$, и в соответствии с (3.9) получаем для $p(x)$ модуль непрерывности (4.11). Заметим, что $k_{0}=k_{0}(\delta) \downarrow 3 k$ при $\delta \rightarrow 0$.

В заключение этого параграфа укажем, что другие примеры соболевских пространств, в которых неплотны гладкие функции, даны в [14], [15], [21], [22]. В приведенном здесь примере детализирована конструкция из [15], что позволило установить некоторые конкретные свойства пространства $W \neq H$. 


\section{§ 5. Пример отсутствия повышенной суммируемости}

5.1. Слабые и вариационные решения. В $\S 2$ для задачи (2.1) введены $W$ - и $H$-решения, а также соответствующие им пространства $W$ и $H$ - самое широкое и самое узкое пространства Соболева-Орлича, связанные с этой задачей. Введем промежуточное пространство $V: H \subseteq V \subseteq W$. Тогда $V$-решение это функция из $V$, для которой тождество (2.3) выполнено на пробных функциях из $V$. Всякое $V$-решение, в том числе $H$ - и $W$-решение, будем называть вариационным. Отметим важную особенность вариационных решений: в определяющее их интегральное тождество можно в качестве пробной функции подставлять само решение и получать энергетическое равенство из (2.4).

Помимо вариационных имеются слабые решения - это функции из $W$, удовлетворяющие уравнению (2.1) в смысле распределений. Ясно, что вариационное решение является и слабым решением, обратное неверно. Однако слабое решение $u$ будет вариационным, если для $u$ выполнено энергетическое равенство из (2.4). В качестве соответствующего промежуточного пространства надо взять $V=H+\{\lambda u\}$.

Существование слабых решений, отличных от вариационных, представляет собой сложный вопрос, не решенный до конца в общем случае. Положительный ответ на него установлен далее при дополнительных условиях, и этим обстоятельством мы воспользуемся при построении основного примера, приведенного ниже.

Часто представляют интерес такие решения, которые получаются с помощью "регуляризации" исходного уравнения. Сопоставим (2.1) регуляризованную задачу

$$
\operatorname{div}\left(\left|\nabla u_{\varepsilon}\right|^{p_{\varepsilon}(x)-2} \nabla u_{\varepsilon}-h\right)=0, \quad x \in \Omega,\left.\quad u_{\varepsilon}\right|_{\partial \Omega}=0,
$$

где $p_{\varepsilon}(x)$ - семейство гладких показателей, удовлетворяющих условию $(2.2)$, таких, что $p_{\varepsilon}(x) \rightarrow p(x)$ п.в. Ввиду регулярности показателя $p_{\varepsilon}(x)$ задаче (5.1) соответствует одно слабое решение. Семейство решений $u_{\varepsilon}(x)$ ограничено в $W^{1, \alpha}(\Omega), u_{\varepsilon} \rightarrow u$ в $W^{1, \alpha}(\Omega)$ (по крайней мере, по подпоследовательности) и по свойству полунепрерывности снизу функционала энергии при слабой сходимости [13]

$$
\liminf _{\varepsilon \rightarrow 0} \int_{\Omega}\left|\nabla u_{\varepsilon}\right|^{p_{\varepsilon}(x)} d x \geqslant \int_{\Omega}|\nabla u|^{p(x)} d x .
$$

Естественно ожидать, что предельная функция $u$ будет слабым решением предельной задачи. Это действительно так при дополнительных предположениях. Приведем некоторые общие факты, доказанные в [23].

ПРЕДЛОЖЕНИЕ 5.1. В случае аппроксимачии сверху, т.е. если $p_{\varepsilon}(x) \geqslant p(x)$ п.в. (или снизу, т.е. если $p_{\varepsilon}(x) \leqslant p(x)$ п.в.), решение задачи (5.1) сходится к Н-решению (или $W$-решению) задачи (2.1).

Будем говорить, что показатель $p(x)$ регулярен вне одной точки $x_{0} \in \Omega$, если из условий $v \in W^{1, p(\cdot)}(\Omega), v \equiv 0$ в окрестности точки $x_{0}$ следует, что $v \in H^{1, p(\cdot)}(\Omega)$. 
ПРЕДЛОЖЕНИЕ 5.2. Пусть $p(x)$ регулярен вне одной точки $x_{0}, u_{\varepsilon}(x)-p e-$ шение задачи $(5.1), u_{\varepsilon}(x) \rightarrow u(x)$ в $W^{1, \alpha}(\Omega)$. Тогда $u(x)$ - слабое решение задачи (2.1). Кроме того, имеет место сходимость плотностей энергии $\left|\nabla u_{\varepsilon}\right|^{p_{\varepsilon}(x)} \rightarrow|\nabla u|^{p(x)}$ в $L^{1}\left(\Omega \backslash B_{\delta}\left(x_{0}\right)\right)$ для любого $\delta \in\left(0, \delta_{0}\right)$.

5.2. Построение примера. Рассмотрим задачу (2.1), предполагая, что $n=2, \Omega=B_{r_{0}}=\left\{|x|<r_{0}\right\}$ - круг достаточно малого радиуса, $p(x)$ - определенный в (4.1) показатель с модулем непрерывности $\omega(t)$, указанным в (4.11). По теореме 4.1 показатель $p(x)$ нерегулярен и согласно предложению 4.5 в уравнении (2.1) можно выбрать правую часть $h \in L^{\infty}(\Omega)$ так, чтобы $u_{1} \neq u_{2}$, где $u_{1}, u_{2}-W$ - и $H$-решения соответственно. Пусть $p_{\varepsilon}^{+}(x)$ и $p_{\varepsilon}^{-}(x)$ - гладкие аппроксимации сверху и снизу для показателя $p(x)$ (см. предложение 5.1) с общим модулем непрерывности $\omega(t)$. Полагая $p_{\varepsilon}(x, t)=t p_{\varepsilon}^{+}(x)+(1-t) p_{\varepsilon}^{-}(x), 0 \leqslant t \leqslant 1$, обозначим соответствующее решение задачи (5.1) через $u_{\varepsilon}(x, t)$.

Найдем линейный функционал $l \in\left(L^{1}(\Omega)\right)^{*}=L^{\infty}(\Omega)$ такой, что $l\left(u_{1}\right) \neq l\left(u_{2}\right)$, и положим $p_{\varepsilon}(x)=p_{\varepsilon}\left(x, t_{\varepsilon}\right)$, где $t_{\varepsilon}$ определено из условия $\lim _{\varepsilon \rightarrow 0} l\left(u_{\varepsilon}\left(\cdot, t_{\varepsilon}\right)\right)=$ $\left(l\left(u_{1}\right)+l\left(u_{2}\right)\right) / 2$. Если $u_{\varepsilon}(x)-$ соответствующее $p_{\varepsilon}(x)$ решение задачи $(5.1)$, то можно считать, что $u_{\varepsilon}(x) \rightarrow u(x)$ в $W^{1, \alpha}(\Omega)$ (в противном случае перейдем к подпоследовательности). Тогда $u \neq u_{1}, u \neq u_{2}$ и $u$ - слабое решение задачи (2.1) (см. предложение 5.2), не являющееся вариационным, так как согласно теореме 4.1 это уравнение не имеет других вариационных решений, кроме $u_{1}$ и $u_{2}$. Поэтому в соотношении (5.2) выполняется строгое неравенство. В противном случае энергетическое равенство для $u_{\varepsilon}$ переходит в пределе в энергетическое равенство для $u$, т.е.

$$
\int_{\Omega} h \cdot \nabla u_{\varepsilon} d x=\int_{\Omega}\left|\nabla u_{\varepsilon}\right|^{p_{\varepsilon}} d x \quad \Longrightarrow \quad \int_{\Omega} h \cdot \nabla u d x=\int_{\Omega}|\nabla u|^{p} d x .
$$

Отсюда следует вариационность решения $u$, что противоречит выбору $u$. Итак, в наших построениях

$$
\lim _{\varepsilon \rightarrow 0} \int_{\Omega}|\nabla u|^{p_{\varepsilon}} d x>\int_{\Omega}|\nabla u|^{p} d x
$$

и, как следствие, семейство $|\nabla u|^{p_{\varepsilon}}$ не может быть слабо компактным в $L^{1}(\Omega)$.

Действительно, в силу предложения $5.2\left|\nabla u_{\varepsilon}\right|^{p_{\varepsilon}} \rightarrow|\nabla u|^{p}$ в $L^{1}\left(\Omega \backslash B_{\delta}(0)\right)$, но при этом сходимость $\left|\nabla u_{\varepsilon}\right|^{p_{\varepsilon}} \rightarrow|\nabla u|^{p}$ в $L^{1}(\Omega)$ исключена неравенством (5.3).

Теперь предположим, что имеет место оценка типа (2.7) с какой-то неограниченно растущей функцией вместо $\ln ^{\delta}(s+2)$ для выбранных показателей $p_{\varepsilon}(x)$. Тогда $\int_{\Omega} \Psi\left(\left|\nabla u_{\varepsilon}\right|^{p_{\varepsilon}}\right) d x<\infty$ для некоторой функции $\Psi(t) \geqslant 0$ такой, что $\lim _{t \rightarrow \infty} \Psi(t) / t=\infty$. По критерию Валле Пуссена [24; гл. VIII, §1] семейство $\left|\nabla u_{\varepsilon}\right|^{p_{\varepsilon}}$ слабо компактно в $L^{1}(\Omega)$, что невозможно, как установлено ранее.

В заключение отметим, что в данном примере модуль непрерывности $\omega(t)$ относится к типу (2.6), причем несложные вычисления показывают, что $k_{0}>$ $3 \alpha / n$ при подходящих значениях параметров $k, \delta, r_{0}$, характеризующих показатель $p(x)$ из (4.1). 


\section{§ 6. Обобщенная лемма Геринга}

6.1. Напомним, что $Q_{r}=Q_{r}\left(x_{0}\right)=[-r / 2, r / 2]^{n}+x_{0}-$ куб со стороной $r>0$ и центром в точке $x_{0} \in \mathbb{R}^{n}$.

Предположим, что $f, g$ - неотрицательные функции, $f, g \in L^{q}\left(\mathbb{R}^{n}\right), q>1$, и найдутся такие $c_{0}>0, r_{0}>0$, что при $r \leqslant r_{0}$

$$
f_{Q_{r}\left(x_{0}\right)} f^{q} d x \leqslant c_{0}\left(f_{Q_{2 r}\left(x_{0}\right)} f d x\right)^{q+b(r)}+f_{Q_{2 r}\left(x_{0}\right)} g^{q} d x \quad \forall x_{0} \in \mathbb{R}^{n} .
$$

Здесь $b(r)$ - некоторая непрерывная неубывающая функция на отрезке $\left[0, r_{0}\right]$, $b(0)=0, b(r) \leqslant 1$. Функцию $f$ считаем нормированной условием $\|f\|_{L^{q}} \leqslant 1$.

ТеОрема 6.1. Если $b(r) \equiv 0$, то справедлива оценка

$$
\int_{\mathbb{R}^{n}} f^{q+\delta} d x \leqslant c\left(1+\int_{\mathbb{R}^{n}} g^{q+\delta} d x\right)
$$

с некоторыми $с>0, \delta>0$, зависящими только от $c_{0}, n, q, r_{0}$.

Это - классический результат, называемый леммой Геринга и имеющий много приложений.

TEOPEMA 6.2. Пусms

$$
b(r)=\frac{k}{\ln (1 / r)}, \quad k>0 .
$$

Тогда справедлива оченка (6.2), при этом константы с >0, $\delta>0$ зависят, помимо прочего, еще и от множителя $k$.

TEOPEMA 6.3. Пусть

$$
b(r)=\frac{k \ln \ln (1 / r)}{\ln (1 / r)}, \quad k>0 .
$$

Тогда при $k \leqslant 1 / n$ справедлива оченка

$$
\int_{\mathbb{R}^{n}} f^{q} \ln ^{\delta}(f+2) d x \leqslant c\left(1+\int_{\mathbb{R}^{n}} g^{q} \ln ^{\delta}(g+2) d x\right)
$$

с некоторыми $\delta>0, c>0$, зависящими только от $c_{0}, n, q, r_{0}$.

Приведенные выше теоремы будут получены как следствие одного общего утверждения, в котором на функцию $b(r)$ накладываются условия технического характера. Положим

$$
a(\lambda)=4^{n+1} c_{0} \lambda^{b\left(\lambda^{-q / n}\right)} .
$$

Tеорема 6.4. Пусть найдется такое $\lambda_{0}$, что

(i) $\lambda_{0}^{q} \geqslant \max \left\{1 / r_{0}^{n}, 1\right\}$;

(ii) функция $s=\lambda / a(\lambda)$ строго возрастает при $\lambda \geqslant \lambda_{0}$; 
(iii) $\int_{s}^{\lambda(s)} \frac{d t}{t(a(t))^{q}} \leqslant C$ npu $s \geqslant s_{0}=\lambda_{0} / a\left(\lambda_{0}\right)$;

(iv) $\lambda a^{\prime}(\lambda) \leqslant a(\lambda) / 2$ npu $\lambda \geqslant \lambda_{0}$.

Тогда справедлива оченка

$$
\int_{\mathbb{R}^{n}} f^{q} \varphi(f) d x \leqslant 4\left[\varphi\left(\lambda_{0}\right)+4^{n+1} \int_{\mathbb{R}^{n}} g^{q} \varphi(4 g) d x\right]
$$

в которой

$$
\varphi(s)= \begin{cases}\exp \left(\theta \int_{s_{0}}^{s} \frac{d t}{t(a(t))^{q}}\right), & s \geqslant s_{0}, \\ 1, & s \in\left[0, s_{0}\right],\end{cases}
$$

а $\theta>0$ удовлетворяет условию

$$
\theta \exp (\theta C) \leqslant \frac{q-1}{4^{n+1}}
$$

Рассмотрим примеры функции $b(r)$, указанные в теоремах 6.1-6.3. Если $b(r) \equiv 0$, то $a(s) \equiv 4^{n+1} c_{0} \equiv a_{0}, \varphi(s)=\left(s / s_{0}\right)^{\delta}, \delta=\theta / a_{0}^{q}$, что дает классическую оценку (6.2). Аналогично, в условиях теоремы 6.2 функция $a(s)$ также постоянна, $a(s) \equiv 4^{n+1} c_{0} \exp (k n / q)$, и из (6.5) следует оценка (6.2).

Теперь обсудим теорему 6.3. Здесь при $k=1 / n$ имеем $(a(s))^{q}=\alpha^{-1} \ln s$, где $\alpha^{-1}=\left(4^{q(n+1)} c_{0}^{q} q\right) / n$,

$$
\int_{s_{0}}^{s} \frac{d t}{t(a(t))^{q}}=\alpha \ln \frac{\ln s}{\ln s_{0}} .
$$

Условия (ii), (iii), (iv) легко проверяются, поэтому выполнена оценка (6.5) с $\varphi(s)=\left(\ln s / \ln s_{0}\right)^{\alpha}$, которую нетрудно переписать в форме оценки (6.4).

6.2. Докажем теорему 6.4. Доказательство разобьем на несколько шагов. $1^{\circ}$. Положим

$$
E(\lambda)=\left\{x \in \mathbb{R}^{n}, f(x)>\lambda\right\} .
$$

Лемма 6.5. Для почти всех $x_{0} \in E(\lambda), \lambda \geqslant \lambda_{0}$, найдется такое $r=$ $r\left(x_{0}, \lambda\right), 0<r<r_{0}$, чmo

$$
f_{Q_{8 r}\left(x_{0}\right)} f^{q} d x \leqslant f_{Q_{r}\left(x_{0}\right)} f^{q} d x=\lambda^{q} .
$$

ДокАЗАТЕЛЬСтво. Для $r \in\left[r_{0}, 8 r_{0}\right]$ выполнено

$$
f_{Q_{r}} f^{q} d x \leqslant \frac{1}{r_{0}^{n}} \int_{\mathbb{R}^{n}} f^{q} d x \leqslant \lambda_{0}^{q} .
$$

С другой стороны, по теореме Лебега

$$
\lim _{r \rightarrow 0} f_{Q_{r}} f^{q} d x>\lambda^{q} \geqslant \lambda_{0}^{q} .
$$

Существование куба $Q_{r}$ со свойством (6.8) следует из непрерывности интеграла $f_{Q_{r}} f^{q} d x$ по $r$. Лемма доказана. 
Из (6.8) получаем, что

$$
\begin{gathered}
\lambda^{q} \leqslant \frac{1}{r^{n}} \int_{\mathbb{R}^{n}} f^{q} d x=\frac{1}{r^{n}}, \quad r \leqslant \lambda^{-q / n}, \quad b(r) \leqslant b\left(\lambda^{-q / n}\right), \\
\lambda^{b(r)} \leqslant \lambda^{b\left(\lambda^{-q / n}\right)}=\frac{a(\lambda)}{c_{0} 4^{n+1}}
\end{gathered}
$$

в силу (6.4) и неравенства $\lambda \geqslant \lambda_{0} \geqslant 1$.

$2^{\circ}$. Оценим интегралы, стоящие в правой части (6.1). Имеем

$$
\begin{aligned}
& \left(f_{Q_{2 r}} f d x\right)^{q+b}=\left(f_{Q_{2 r}} f d x\right)^{q-1+b} \cdot f_{Q_{2 r}} f d x \leqslant\left(f_{Q_{2 r}} f^{q} d x\right)^{(q-1+b) / q} \cdot f_{Q_{2 r}} f d x \\
& \leqslant\left(4^{n} f_{Q_{8 r}} f^{q} d x\right)^{(q-1+b) / q} \cdot f_{Q_{2 r}} f d x \leqslant 4^{n} \lambda^{q-1+b} f_{Q_{2 r}} f d x \\
& \leqslant \frac{a(\lambda) \lambda^{q-1}}{4 c_{0}} f_{Q_{2 r}} f d x
\end{aligned}
$$

где использованы неравенства (6.8), (6.9) и $0 \leqslant b \leqslant 1$. Далее,

$$
f_{Q_{2 r}} f d x \leqslant \lambda \eta+\frac{1}{\left|Q_{2 r}\right|} \int_{Q_{2 r} \cap E(\eta \lambda)} f d x, \quad \eta \in(0,1),
$$

и положив $\eta=1 /(a(\lambda))$, выводим из $(6.10)$

$$
c_{0}\left(f_{Q_{2 r}} f d x\right)^{q+b} \leqslant \frac{\lambda^{q}}{4}+\frac{a(\lambda) \lambda^{q-1}}{4\left|Q_{2 r}\right|} \int_{Q_{2 r} \cap E(s)} f d x, \quad s=\frac{\lambda}{a(\lambda)} .
$$

Кроме того, если $D(\lambda)=\left\{x \in \mathbb{R}^{n}, g(x)>\lambda\right\}$, то

$$
f_{Q_{2 r}} g^{q} d x \leqslant \frac{1}{4} \lambda^{q}+\frac{1}{\left|Q_{2 r}\right|} \int_{Q_{2 r} \cap D(\lambda / 4)} g^{q} d x .
$$

Теперь из (6.8), (6.1), (6.11), (6.12) получаем, что

$$
\begin{gathered}
\lambda^{q}=f_{Q_{r}} f^{q} d x \leqslant \frac{\lambda^{q}}{2}+\frac{a(\lambda) \lambda^{q-1}}{4\left|Q_{2 r}\right|} \int_{Q_{2 r} \cap E(s)} f d x+\frac{1}{\left|Q_{2 r}\right|} \int_{Q_{2 r} \cap D(\lambda / 4)} g^{q} d x \\
f_{Q_{8 r}} f^{q} d x \leqslant f_{Q_{r}} f^{q} d x \leqslant \frac{a(\lambda)}{2} \frac{\lambda^{q-1}}{\left|Q_{2 r}\right|} \int_{Q_{2 r} \cap E(s)} f d x+\frac{2}{\left|Q_{2 r}\right|} \int_{Q_{2 r} \cap D(\lambda / 4)} g^{q} d x \\
\int_{Q_{8 r}} f^{q} d x \leqslant 4^{n} a(\lambda) \lambda^{q-1} \int_{Q_{2 r} \cap E(s)} f d x+4^{n+1} \int_{Q_{2 r} \cap D(\lambda / 4)} g^{q} d x
\end{gathered}
$$

Итак, для почти всех $x_{0} \in E(\lambda), \lambda \geqslant \lambda_{0}$, существует куб со стороной $r=$ $r\left(x_{0}, \lambda\right)$, для которого выполнено неравенство (6.13). По теореме Витали о покрытиях найдется счетное семейство непересекающихся кубов $Q_{2 r_{j}}$ такое, что

$$
E(\lambda) \subset \bigcup Q_{8 r_{j}} .
$$


В этом случае из (6.13) следует, что

$$
\int_{E(\lambda)} f^{q} d x \leqslant 4^{n} \lambda^{q-1} a(\lambda) \int_{E(s)} f d x+4^{n+1} \int_{D(\lambda / 4)} g^{q} d x, \quad s=\frac{\lambda}{a(\lambda)} .
$$

$3^{\circ}$. Ниже используем следующие соотношения:

$$
\begin{gathered}
J \equiv \int_{E\left(\lambda_{0}\right)} f^{q} \varphi(f) d x=-\int_{\lambda_{0}}^{\infty} \varphi(\lambda) d_{\lambda}\left(\int_{E(\lambda)} f^{q} d x\right) \\
=\int_{\lambda_{0}}^{\infty} \varphi^{\prime}(\lambda) \int_{E(\lambda)} f^{q} d x d \lambda+\varphi\left(\lambda_{0}\right) \int_{E\left(\lambda_{0}\right)} f^{q} d x \\
\int_{E\left(\lambda_{0}\right)} f \psi(f) d x=\int_{\lambda_{0}}^{\infty} \psi^{\prime}(\lambda) \int_{E(\lambda)} f d x d \lambda+\psi\left(\lambda_{0}\right) \int_{E\left(\lambda_{0}\right)} f d x .
\end{gathered}
$$

Теперь из $(6.15),(6.14)$ получим, что

$$
\begin{aligned}
J \leqslant 4^{n} & \int_{\lambda_{0}}^{\infty} \varphi^{\prime}(\lambda) a(\lambda) \lambda^{q-1} \int_{E(s)} f d x d \lambda+4^{n+1} \int_{\lambda_{0}}^{\infty} \varphi^{\prime}(\lambda) \int_{D(\lambda / 4)} g^{q} d x d \lambda \\
& +\varphi\left(\lambda_{0}\right) \int_{\mathbb{R}^{n}} f^{q} d x \leqslant J_{1}+J_{2}+\varphi\left(\lambda_{0}\right),
\end{aligned}
$$

поскольку $\|f\|_{L^{q}} \leqslant 1$. Изучим слагаемое

$$
J_{1}=4^{n} \int_{s_{0}}^{\infty} \lambda^{\prime} \varphi^{\prime}(\lambda) a(\lambda) \lambda^{q-1} \int_{E(s)} f d x d s, \quad \lambda=\lambda(s) .
$$

Положим

$$
\psi^{\prime}(s)=\lambda^{\prime} \varphi^{\prime}(\lambda) a(\lambda) \lambda^{q-1}, \quad \lambda=\lambda(s) .
$$

Убедимся, что выполнено неравенство

$$
\psi(t)=\int_{s_{0}}^{t} \psi^{\prime}(s) d s \leqslant \frac{\varphi(t) t^{q-1}}{2 \cdot 4^{n}}, \quad t \geqslant s_{0} .
$$

Действительно, используя соотношение $\lambda=s a(\lambda(s))$ и определение (6.6) функции $\varphi$, запишем

$$
\psi^{\prime}(s)=\lambda^{\prime} \varphi^{\prime}(\lambda) a^{q}(\lambda) \lambda \frac{s^{q-2}}{a(\lambda)}=\theta \lambda^{\prime} \frac{\varphi(\lambda)}{a(\lambda)} s^{q-2} .
$$

Из условия (iv) теоремы 6.4 имеем

$$
s^{\prime}(\lambda)=\frac{1}{a(\lambda)}-\frac{\lambda a^{\prime}(\lambda)}{(a(\lambda))^{2}} \geqslant \frac{1}{2 a(\lambda)}, \quad \lambda^{\prime}(s)=\frac{1}{s^{\prime}(\lambda(s))} \leqslant 2 a(\lambda(s)), \quad s \geqslant s_{0} .
$$

Кроме того, по условию (iii)

$$
\varphi(\lambda(s)) \leqslant e^{\theta C} \varphi(s), \quad s \geqslant s_{0},
$$

поэтому

$$
\psi^{\prime}(s) \leqslant 2 \theta e^{\theta C} \varphi(s) s^{q-2}, \quad \psi(t) \leqslant 2 \theta e^{\theta C} \varphi(t) \int_{s_{0}}^{t} s^{q-2} d s \leqslant 2 \theta e^{\theta C} \frac{\varphi(t) t^{q-1}}{q-1}
$$

и неравенство (6.18) выполнено, если только $\theta$ удовлетворяет условию (6.7). 
В результате по формуле (6.16) получаем, что

$$
\begin{aligned}
J_{1} & =4^{n} \int_{s_{0}}^{\infty} \psi^{\prime}(s) \int_{E(s)} f d x d s \leqslant 4^{n} \int_{E\left(s_{0}\right)} f \psi(f) d x \\
& \leqslant \frac{1}{2} \int_{E\left(s_{0}\right)} f^{q} \varphi(f) d x \leqslant \frac{1}{2} J+\frac{1}{2} \int_{\mathbb{R}^{n} \backslash E\left(\lambda_{0}\right)} f^{q} \varphi(f) d x \leqslant \frac{1}{2} J+\frac{1}{2} \varphi\left(\lambda_{0}\right) .
\end{aligned}
$$

Рассмотрим слагаемое $J_{2}$ (см. (6.17)). По формуле (6.15) (для $g$ вместо $f$ ) имеем

$$
\begin{aligned}
J_{2} & =4^{n+1} \int_{\lambda_{0} / 4} 4 \varphi^{\prime}(4 \lambda) \int_{D(\lambda)} g^{q} d x d \lambda \leqslant 4^{n+1} \int_{D\left(\lambda_{0} / 4\right)} g^{q} \varphi(4 g) d x \\
& \leqslant 4^{n+1} \int_{\mathbb{R}^{n}} g^{q} \varphi(4 g) d x .
\end{aligned}
$$

Собирая все оценки, получим

$$
\begin{aligned}
J & \leqslant \frac{1}{2} J+J_{2}+\frac{3}{2} \varphi\left(\lambda_{0}\right), \quad J \leqslant 3 \varphi\left(\lambda_{0}\right)+2 J_{2}, \\
\int_{\mathbb{R}^{n}} f^{q} \varphi(f) d x & =J+\int_{\mathbb{R}^{n} \backslash E\left(\lambda_{0}\right)} f^{q} \varphi(f) d x \leqslant J+\varphi\left(\lambda_{0}\right) \leqslant 2 J_{2}+4 \varphi\left(\lambda_{0}\right),
\end{aligned}
$$

что дает оценку (6.5), если учесть неравенство (6.19). Теорема 6.4 доказана.

ВЫВод теОРем 1.1-1.3. Из доказательства теоремы 6.4 видно, что выполнение неравенства (6.1) достаточно требовать лишь для точек $x_{0}$ таких, что $f\left(x_{0}\right) \geqslant \lambda_{0}$ при некотором $\lambda_{0} \geqslant 1$. Если $f \equiv 0$ вне области $\Omega$, то такие точки находятся заведомо в $\Omega$. Тогда в предположениях теорем 1.1-1.3 (см. (1.10)) можно получить оценку (6.5), которая в каждом из трех случаев, как показано выше, имеет вид (1.11) или (1.12).

\section{§7. Приложение: о пространствах Соболева-Орлича}

В последние годы много внимания уделялось вариационным задачам для интегральных функционалов вида

$$
\int_{\Omega}|\nabla u(x)|^{p(x)} d x,
$$

где измеримый в области $\Omega \subset \mathbb{R}^{n}$ показатель $p(x)$ удовлетворяет условию $(2.2)$. С такими функционалами естественно связаны пространства Соболева-Орлича, и многие вопросы общей теории получают более адекватный ответ в терминах этих пространств.

Приведем некоторые сведения из теории пространств Соболева-Орлича [25], [26].

7.1. Через $L^{p(\cdot)}(\Omega)$ обозначим множество всех измеримых на $\Omega$ функций $f(x)$ таких, что

$$
A_{p}(f)=\int_{\Omega}|f(x)|^{p(x)} d x<\infty .
$$


Класс $L^{p(\cdot)}(\Omega)$ будет банаховым (рефлексивным (см. лемму 7.4) и сепарабельным) пространством, если норму $\|\cdot\|_{L^{p(\cdot)(\Omega)}}=\|\cdot\|_{L^{p(\cdot)}}$ определить равенством

$$
\|f\|_{L^{p(\cdot)}}=\inf \left\{\lambda>0, A_{p}\left(\lambda^{-1} f\right) \leqslant 1\right\} .
$$

Заметим, что инфимум в (7.1) достигается, если $A_{p}(f)>0$; кроме того,

$$
\|f\|_{L^{p(\cdot)}}=\lambda>0 \quad \Longleftrightarrow \quad A_{p}\left(\lambda^{-1} f\right)=1 .
$$

Отсюда легко получить соотношения

$$
\begin{gathered}
\|f\|_{L^{p(\cdot)}} \leqslant 1 \quad \Longleftrightarrow \quad A_{p}(f) \leqslant 1, \\
\|f\|_{L^{p(\cdot)}} \geqslant 1 \quad \Longleftrightarrow \quad\|f\|_{L^{p(\cdot)}}^{\alpha} \leqslant A_{p}(f) \leqslant\|f\|_{L^{p(\cdot)}}^{\beta}, \\
\|f\|_{L^{p(\cdot)}} \leqslant 1 \quad \Longleftrightarrow\|f\|_{L^{p(\cdot)}}^{\beta} \leqslant A_{p}(f) \leqslant\|f\|_{L^{p(\cdot)}}^{\alpha}, \\
f_{n} \rightarrow f \text { в } L^{p(\cdot)} \quad \Longleftrightarrow \quad A_{p}\left(f_{n}-f\right) \rightarrow 0 .
\end{gathered}
$$

Отметим следующие полезные факты.

Лемма 7.1. Пусть ограниченная в $L^{p(\cdot)}(\Omega)$ последовательность $f_{\varepsilon}$ такова, что $f_{\varepsilon}(x) \rightarrow f(x)$ почти всюду на $\Omega$. Тогда $f \in L^{p(\cdot)}(\Omega)$ u $f_{\varepsilon} \rightarrow f$ в $L^{p(\cdot)}(\Omega)$.

ЛЕмма 7.2. Если $\Omega$ - ограниченная область, то имеют место непрерывные вложения $L^{\beta}(\Omega) \subset L^{p(\cdot)}(\Omega) \subset L^{\alpha}(\Omega)$ с оценками норм

$$
\begin{aligned}
\|f\|_{L^{\alpha}(\Omega)} & \leqslant(1+|\Omega|)^{1 / \alpha}\|f\|_{L^{p(\cdot)}(\Omega)}, \\
\|f\|_{L^{p(\cdot)}(\Omega)} & \leqslant(1+|\Omega|)^{1 / \beta^{\prime}}\|f\|_{L^{\beta}(\Omega)}, \quad \beta^{\prime}=\frac{\beta}{\beta-1} .
\end{aligned}
$$

ДокАЗАТЕЛЬСтво. Из (7.2) имеем

$$
\int_{\Omega}\left|\lambda^{-1} f\right|^{\alpha} d x \leqslant \int_{\Omega}\left(\left|\lambda^{-1} f\right|^{p}+1\right) d x=1+|\Omega|, \quad \int_{\Omega}|f|^{\alpha} d x \leqslant \lambda^{\alpha}(1+|\Omega|),
$$

что дает оценку (7.5). Оценка (7.6) будет получена далее по двойственности.

Класс $L^{p(\cdot)}(\Omega)$ введен в основополагающей работе Орлича [27]. В этой работе доказано, что $L^{p(\cdot)}(\Omega)$ есть банахово пространство относительно "нормы Орлича"

$$
\mu_{p(\cdot)}(f) \equiv \sup \left\{\left.\int_{\Omega} f g d x\left|\int_{\Omega}\right| g\right|^{p^{\prime}} d x \leqslant 1\right\},
$$

где $p^{\prime}(x)=p(x) /(p(x)-1)$ - сопряженный показатель. Как показывает следующая лемма, норма Орлича эквивалентна введенной ранее норме (7.1), называемой нормой Люксембурга.

ЛЕмма 7.3. Имеют место неравенства

$$
\|f\|_{L^{p(\cdot)}} \leqslant \mu_{p(\cdot)}(f) \leqslant\left(\frac{1}{\alpha}+\frac{1}{\beta^{\prime}}\right)\|f\|_{L^{p(\cdot)}} \leqslant 2\|f\|_{L^{p(\cdot)}} .
$$


ДоказАтЕЛЬСтво. Пусть $\lambda=\|f\|_{L^{p(\cdot)}}, g=\left|\lambda^{-1} f\right|^{p-2} \lambda^{-1} f$. Тогда $|g|^{p^{\prime}}=$ $\left|\lambda^{-1} f\right|^{p}$ и поэтому $\int_{\Omega}|g|^{p^{\prime}} d x=1$. Отсюда

$$
\mu_{p(\cdot)}(f) \geqslant \int_{\Omega} f g d x=\lambda \int_{\Omega}\left|\lambda^{-1} f\right|^{p} d x=\lambda=\|f\|_{L^{p(\cdot)}}
$$

в силу свойства (7.2). Этим доказано левое неравенство (7.8). Правое неравенство есть следствие неравенства Юнга

$$
f g \leqslant \frac{|f|^{p}}{p}+\frac{|g|^{p^{\prime}}}{p^{\prime}} \leqslant \frac{|f|^{p}}{\alpha}+\frac{|g|^{p^{\prime}}}{\beta^{\prime}} .
$$

Действительно, можно считать, что $\|f\|_{L^{p(\cdot)}}=1$ и, таким образом, (см. (7.2)) $\int_{\Omega}|f|^{p} d x=1$. Если к тому же $\int_{\Omega}|g|^{p^{\prime}} d x \leqslant 1$, то

$$
\int_{\Omega} f g d x \leqslant \frac{1}{\alpha}+\frac{1}{\beta^{\prime}} \Longrightarrow \mu_{p(\cdot)}(f) \leqslant \frac{1}{\alpha}+\frac{1}{\beta^{\prime}} .
$$

Лемма доказана.

В качестве следствия получим аналог неравенства Гёльдера

$$
\int_{\Omega} f g d x \leqslant 2\|f\|_{L^{p(\cdot)}}\|g\|_{L^{p^{\prime}(\cdot)}} .
$$

Действительно, пусть $\lambda=\|g\|_{p^{\prime}(\cdot)}$. Тогда $\int_{\Omega}\left|\lambda^{-1} g\right|^{p^{\prime}} d x=1$ и из (7.7) имеем

$$
\int_{\Omega} f \lambda^{-1} g d x \leqslant \mu_{p(\cdot)}(f) \leqslant 2\|f\|_{L^{p(\cdot)}}, \quad \int_{\Omega} f g d x \leqslant 2\|f\|_{L^{p(\cdot)}}\|g\|_{L^{p(\cdot)}},
$$

и неравенство (7.9) доказано.

В качестве другого следствия выведем оценку (7.6). Имеем (см. (7.7), (7.3))

$$
\begin{aligned}
\|f\|_{L^{p(\cdot)}} & \leqslant \mu_{p(\cdot)}(f)=\sup \left\{\int_{\Omega} f g d x \mid\|g\|_{L^{p^{\prime}(\cdot)}} \leqslant 1\right\} \\
& \leqslant \sup \left\{\int_{\Omega} f g d x \mid\|g\|_{L^{\beta^{\prime}}} \leqslant(1+|\Omega|)^{1 / \beta^{\prime}}\right\},
\end{aligned}
$$

где использован аналог оценки $(7.5)$ для пространств $L^{p^{\prime}(\cdot)}(\Omega) \subset L^{\beta^{\prime}}(\Omega)$. Отсюда по неравенству Гёльдера с показателями $\beta$ и $\beta^{\prime}$ следует искомая оценка (7.6).

ЛЕмма 7.4. Пространство $L^{p(\cdot)}(\Omega)$ рефллексивно, а сопряжсеным $к$ нему служст пространство $L^{p^{\prime}(\cdot)}(\Omega)$. Другими словами, всякий непрерывный на $L^{p(\cdot)}(\Omega)$ функиионал $l=l(f)$ имеет вид

$$
l(f)=\int_{\Omega} f g d x, \quad g \in L^{p^{\prime}(\cdot)}(\Omega) .
$$

ДокАЗАТЕльство. Так как $L^{\beta}(\Omega) \subset L^{p(\cdot)}(\Omega)$, то функционал $l$ непрерывен на $L^{\beta}(\Omega)$ и допускает представление

$$
l(f)=\int_{\Omega} f g d x, \quad f \in L^{\beta}(\Omega),
$$


где $g$ - некоторый элемент из $L^{\beta^{\prime}}(\Omega)$. Убедимся в том, что $g \in L^{p^{\prime}(\cdot)}(\Omega)$. Положив

$$
g_{N}(x)=\left\{\begin{array}{ll}
g(x), & \text { если }|g(x)| \leqslant N \\
0, & \text { если }|g(x)|>N,
\end{array} \quad f_{N}(x)=\left|g_{N}\right|^{p^{\prime}-2} g_{N},\right.
$$

в силу непрерывности функционала $l$ на $L^{p(\cdot)}$ получим, что

$$
l\left(f_{N}\right)=\int_{\Omega} f_{N} g d x=\int_{\Omega}\left|g_{N}\right|^{p^{\prime}} d x \leqslant c\left\|f_{N}\right\|_{L^{p(\cdot)}} .
$$

Тогда из (7.4) имеем

$$
\int_{\Omega}\left|g_{N}\right|^{p^{\prime}} d x \leqslant c\left(\int_{\Omega}\left|f_{N}\right|^{p} d x\right)^{1 / \gamma}=c\left(\int_{\Omega}\left|g_{N}\right|^{p^{\prime}} d x\right)^{1 / \gamma},
$$

где $\gamma=\alpha$, если $\left\|f_{N}\right\|_{L^{p(\cdot)}} \geqslant 1$, и $\gamma=\beta$ в противном случае. Отсюда по лемме Фату

$$
\int_{\Omega}\left|g_{N}\right|^{p^{\prime}} d x \leqslant c^{\gamma^{\prime}}, \quad \int_{\Omega}|g|^{p^{\prime}} d x \leqslant c^{\gamma^{\prime}} .
$$

Лемма доказана.

Отметим, что если на $L^{p(\cdot)}(\Omega)$ задать норму Люксембурга (7.1) (соответственно норму Орлича $(7.7))$, то на пространстве $L^{p^{\prime}(\cdot)}(\Omega)$ как сопряженном к $L^{p(\cdot)}(\Omega)$, возникает норма Орлича (соответственно Люксембурга). Это ясно из свойства (7.3), дающего описание единичного шара в норме (7.1) и определения норм.

7.2. Предположим, что область $\Omega$ ограничена и имеет липшицеву границу. Определим пространство Соболева-Орлича

$$
\begin{gathered}
W^{1, p(\cdot)}(\Omega)=\left\{u \in W^{1,1}(\Omega), \int_{\Omega}|\nabla u|^{p(x)} d x<\infty\right\}, \\
\|u\|_{W^{p(\cdot)}(\Omega)}=\|u\|_{L^{1}(\Omega)}+\|\nabla u\|_{L^{p(\cdot)}(\Omega)},
\end{gathered}
$$

где $\|\nabla u\|_{L^{p(\cdot)(\Omega)}}$ означает норму функции $|\nabla u|$ в $L^{p(\cdot)}(\Omega)$. Определим также связанное с задачей Дирихле пространство

$$
\begin{gathered}
W_{0}^{1, p(\cdot)}(\Omega)=\left\{u \in W_{0}^{1,1}(\Omega), \int_{\Omega}|\nabla u|^{p} d x<\infty\right\}, \\
\|u\|_{W_{0}^{p(\cdot)}(\Omega)}=\|\nabla u\|_{L^{p(\cdot)}(\Omega)} .
\end{gathered}
$$

Лемма 7.5. Множество $W_{0}^{1, p(\cdot)}(\Omega) \cap L^{\infty}(\Omega)$ плотно в $W_{0}^{1, p(\cdot)}(\Omega)$.

ДокАЗАТЕЛЬСтво. Для срезки

$$
u^{(k)}= \begin{cases}u(x), & \text { если }|u(x)| \leqslant k \\ \pm k, & \text { если }|u(x)|>k\end{cases}
$$
имеем $\left|\nabla u^{(k)}\right| \leqslant|\nabla u|$ и по теореме Лебега $\lim _{k \rightarrow \infty} \int_{\Omega}\left|\nabla u-\nabla u^{(k)}\right|^{p} d x \rightarrow 0$. Лемма
доказана. 
Отметим, что вложение $W_{0}^{1, p(\cdot)}(\Omega) \subset L^{p(\cdot)}(\Omega)$, вообще говоря, не имеет места без дополнительных условий на показатель.

ЛЕмма 7.6. Если показатель р непрерывен в $\bar{\Omega}$, то имеет место компактное вложение $W^{1, p(\cdot)}(\Omega) \subset L^{p(\cdot)}(\Omega)$.

ДокАзАтЕльство. Достаточно проверить указанное вложение в каждой подобласти $\Omega \cap B$, где $B$ - шар малого радиуса. Считаем, что

$$
p_{2}=\max _{\Omega \cap B} p(x) \leqslant \frac{n+1}{n} p_{1}, \quad p_{1}=\min _{\Omega \cap B} p(x) .
$$

В этом случае $W^{1, p(\cdot)}(\Omega \cap B) \subset W^{1, p_{1}}(\Omega \cap B) \subset L^{p_{2}}(\Omega \cap B) \subset L^{p(\cdot)}(\Omega \cap B)$; при этом все вложения непрерывны, а второе из них компактно по теореме вложения Соболева. Отсюда следует утверждение леммы.

ЛЕмма 7.7. Пусть показатель р непрерывен в $\bar{\Omega}, l$ - непрерывный функционал в $L^{1}(\Omega), l(1)>0$. Тогда справедлива оценка

$$
\|u-l(u)\|_{L^{p(\cdot)}(\Omega)} \leqslant c_{0}\|\nabla u\|_{L^{p(\cdot)}(\Omega)},
$$

причем постоянная $c_{0}$ зависит от $\Omega$, модуля непрерывности показателя $p$ и выбора функционала $l$.

Оценка (7.10) получается из компактности вложения $W^{1, p(\cdot)}(\Omega) \subset L^{p(\cdot)}(\Omega)$ стандартными рассуждениями от противного. Полезно эту оценку записать в более явной форме, используя соотношения (7.4), а именно,

$$
\int_{\Omega}|u-l(u)|^{p} d x \leqslant\left(c_{0}+1\right)\left[\left(\int_{\Omega}|\nabla u|^{p} d x\right)^{\beta / \alpha}+1\right] .
$$

Обратим внимание на то, что оценка (7.11) отличается от классического неравенства Пуанкаре, которое имеет место при постоянном $p$. Примеры показывают [28], что в общем случае в правой части (7.11) нельзя избавиться ни от показателя $\beta / \alpha$, ни от постоянного слагаемого. Однако указанные упрощения возможны при определенных дополнительных предположениях на показатель $p$. Например, справедлива использованная при выводе леммы 4.3 следующая

Лемма 7.8. Пусть $n=1, I=(a, b), I_{0}=\left(a_{0}, b_{0}\right),-\infty<a \leqslant a_{0}<b_{0} \leqslant b<\infty$, и пусть на I определена измеримая функиия $p(t)$ такая, что:

1) $\left.p\right|_{I_{0}} \equiv p_{0}=\mathrm{const}$;

2) $1 \leqslant p(t) \leqslant p_{0}, \quad t \in I$

3) $p(t)$ монотонна на $\left(a, a_{0}\right) u\left(b_{0}, b\right)$.

Тогда

$$
\int_{I}\left|u(t)-f_{I_{0}} u d t\right|^{p(t)} d t \leqslant c_{0}+c_{0} \int_{I}\left|u^{\prime}(t)\right|^{p(t)} d t \quad \forall u \in C^{1}(I),
$$

где константа с зависит лищь от величин $b-a$ и $p_{0}$. 
ДокАЗАТЕльство. Достаточно доказать неравенство (7.12) для отрезка $I=$ $(0,1)$ и функции $u$ со средним $f_{I_{0}} u d t=0$. В этом случае найдется точка $t_{0} \in I_{0} \subset(0,1)$ такая, что $u\left(t_{0}\right)=0$, а $p(t)$ не убывает на $\left(0, t_{0}\right)$ и не возрастает на $\left(t_{0}, 1\right)$. Отсюда для $t \in\left(t_{0}, 1\right)$ имеем

$$
|u(t)|^{p(t)} \leqslant \int_{t_{0}}^{t}\left|u^{\prime}(s)\right|^{p(t)} d s \leqslant \int_{t_{0}}^{t}\left(\left|u^{\prime}(s)\right|^{p(s)}+1\right) d s \leqslant 1+\int_{t_{0}}^{1}\left|u^{\prime}(s)\right|^{p(s)} d s
$$

и после интегрирования по $t \in\left(t_{0}, 1\right)$

$$
\int_{t_{0}}^{1}|u(t)|^{p(t)} d t \leqslant 1-t_{0}+\int_{t_{0}}^{1}\left|u^{\prime}(t)\right|^{p(t)} d t
$$

Аналогично показываем, что

$$
\int_{0}^{t_{0}}|u(t)|^{p(t)} d t \leqslant t_{0}+\int_{0}^{t_{0}}\left|u^{\prime}(t)\right|^{p(t)} d t
$$

Суммируя оценки, получаем

$$
\int_{0}^{1}|u(t)|^{p(t)} d t \leqslant 1+\int_{0}^{1}\left|u^{\prime}(t)\right|^{p(t)} d t
$$

В случае отрезка $I=(a, b)$ общего вида с помощью замены $t=a+(b-a) \tau$, где $\tau \in(0,1)$, из $(7.13)$ выводим

$$
\int_{a}^{b}|u(t)|^{p(t)} d t \leqslant b-a+\max \left(1,(b-a)^{p_{0}}\right) \int_{a}^{b}\left|u^{\prime}(t)\right|^{p(t)} d t
$$

откуда следует (7.12). Лемма доказана.

\section{Список литературы}

[1] A. P. Calderon, A. Zygmund, "On the existence of certain singular integrals", Acta Math., 88:1 (1952), 85-139.

[2] N. G. Meyers, "An $L^{p}$-estimate for the gradient of solutions of second order elliptic divergence equations", Ann. Scuola Norm. Sup. Pisa (3), 17 (1963), 189-206.

[3] Б. В. Боярский, "Обобщенные решения системы дифференциальных уравнений первого порядка эллиптического типа с разрывными коэффициентами", Матем. сб., 43(85):4 (1957), 451-503.

[4] F.W. Gehring, "The $L^{p}$-integrability of the partial derivatives of a quasiconformal mapping", Acta Math., 130:1 (1973), 265-277.

[5] M. Giaquinta, G. Modica, "Regularity results for some classes of higher order non linear elliptic systems", J. Reine Angew. Math., 311-312 (1979), 145-169.

[6] M. Giaquinta, Multiple integrals in the calculus of variations and nonlinear elliptic systems, Ann. of Math. Stud., 105, Princeton Univ. Press, Princeton, NJ, 1983.

[7] M. Giaquinta, Introduction to regularity theory for nonlinear elliptic systems, Lectures Math. ETH Zürich, Birkhäuser, Basel, 1993. 
[8] И.В. Скрыпник, Методы исследования нелинейных эллиптических граничных задач, Наука, М., 1990; англ. пер.: I. V. Skrypnik, Methods for analysis of nonlinear elliptic boundary value problems, Transl. Math. Monogr., 139, Amer. Math. Soc., Providence, RI, 1994.

[9] V. V. Zhikov, "On some variational problems", Russian J. Math. Phys., 5:1 (1997), 105-116.

[10] В. В. Жиков, “Об оценках типа Мейерса для решений нелинейного уравнения Стокса", Дифферени. уравнения, 33:1 (1997), 107-114; англ. пер.: V. V. Zhikov, "Meyers type estimates for the solution of a nonlinear Stokes system", Differential Equations, 33:1 (1997), 108-115.

[11] В. В. Жиков, С.Е. Пастухова, "О лемме Геринга", Докл. РАН, 419:4 (2008), 443-448; англ. пер.: V. V. Zhikov, S. E. Pastukhova, "On Gehring's lemma", Dokl. Math., 77:2 (2008), 243-248.

[12] Ж.-Л. Лионс, Некоторые методы решения нелинейных краевых задач, Мир, М., 1972; пер. с фр. J.-L. Lions, Quelques méthodes de résolution des problèmes aux limites non linéaires, Dunod, de Gruyter, Paris, 1969.

[13] В.В.Жиков, "Разрешимость трехмерной задачи о термисторе", Дифференциальные уравнения и динамические системы, Сборник статей, Тр. МИАН, 261, Наука, M., 2008, 101-114; англ. пер.: V. V. Zhikov, "Solvability of the three-dimensional thermistor problem", Proc. Steklov Inst. Math., 261:1 (2008), 98-111.

[14] В. В. Жиков, "Об эффекте Лаврентьева", Докл. РАН, 345:1 (1995), 10-14; англ. пер.: V. V. Zhikov, "On Lavrent'ev's effect", Russian Acad. Sci. Dokl. Math., 52:3 (1995), 325-329.

[15] В.В.Жиков, "О плотности гладких функций в пространстве Соболева-Орлича", Краевые задачи математической физики и смежные вопросы теории функuий. 35, Зап. научн. сем. ПОМИ, 310, ПОМИ, СПб., 2004, 67-81; англ. пер.: V.V. Zhikov, "Density of smooth functions in Sobolev-Orlicz spaces", J. Math. Sci. (N. Y.), 132:3 (2006), 285-294.

[16] E. Acerbi, G. Mingione, "Regularity results for a class of functionals with non-standard growth", Arch. Ration. Mech. Anal., 156:2 (2001), 121-140.

[17] Ю.А. Алхутов, "Неравенство Харнака и гёльдеровость решений нелинейных уравнения с нестандартным условием роста", Дифферени. уравнения, 33:12 (1997), 1651-1660; англ. пер.: Yu. A. Alkhutov, "The Harnack inequality and the Holder property of solutions of nonlinear elliptic equations with a nonstandard growth condition", Differential Equations, 33:12 (1997), 1653-1663.

[18] L. Diening, "Maximal function on Musielak-Orlicz spaces and generalized Lebesgue spaces", Bull. Sci. Math., 129:8 (2005), 657-700.

[19] E. Acerbi, G. Mingione, "Regularity results for stationary electro-rheological fluids", Arch. Ration. Mech. Anal., 164:3 (2002), 213-259.

[20] Д. Киндерлерер, Г. Стампаккья, Введение в вариачионные неравенства и их приложения, Мир, М., 1983; пер. с англ.: D. Kinderlehrer, G. Stampacchia, An introduction to variational inequalities and their applications, Pure Appl. Math., 88, Academic Press, New York-London, 1980.

[21] В. В. Жиков, "Вопросы сходимости, двойственности и усреднения для функционалов вариационного исчисления", Изв. АН СССР. Сер. матем., 47:5 (1983), 961-998; англ. пер.: V. V. Zhikov, "Questions of convergence, duality, and averaging for functionals of the calculus of variations", Math. USSR-Izv., 23:2 (1984), 243-276.

[22] В. В. Жиков, "О переходе к пределу в нелинейных вариационных задачах", Maтем. сб., 183:8 (1992), 47-84; англ. пер.: V. V. Zhikov, "On passage to the limit in nonlinear variational problems", Russian Acad. Sci. Sb. Math., 76:2 (1993), 427-459.

[23] В.В. Жиков, "К технике предельного перехода в нелинейных эллиптических уравнениях", Функи. анализ и его приложения, 43:2 (2009) (в печати). 
[24] И. Экланд, Р. Темам, Выпуклый анализ и вариационные проблемы, Мир, М., 1979; пер. с англ.: I. Ekeland, R. Temam, Convex analysis and variational problems, Studies in Mathematics and its Applications, 1, North-Holland, Amsterdam-Oxford; Elsevier, New York, 1976.

[25] D.E. Edmunds, J. Rákosnik, "Sobolev embeddings with variable exponent", Studia Math., 143:3 (2000), 267-293.

[26] X. Fan, J. Shen, D. Zhao, "Sobolev embedding theorems for spaces $W^{k, p(x)}(\Omega)$ ", J. Math. Anal. Appl., 262:2 (2001), 749-760.

[27] W. Orlicz, "Über konjugierte Exponentenfolgen", Stud. Math., 3 (1931), 200-211.

[28] F.-Y. Maeda, "Poincare type inequality and an integrability result for variable exponent", JIPAM. J. Inequal. Pure Appl. Math., 9:3 (2008), article 68.

\section{В. В. Жиков (V. V. Zhikov)}

Владимирский государственный педагогический университет

E-mail: zhikov@vgpu.vladimir.ru

\section{C. Е. Пастухова (S. E. Pastukhova)}

Московский государственный институт радиотехники, электроники и автоматики (технический университет)

E-mail: leonowmw@cs.msu.su
Поступила в редакцию 26.03.2008 и 17.06 .2008 